\title{
INFLUENCE DES PHÉNOMĖNES DE COUP DE BÉLIER SUR LE RÉGLAGE DE LA VITESSE DES TURBINES HYDRAULIQUES
}

\section{THE INFLUENCE OF WATER HAMMER EFFECTS ON THE GOVERNING OF HYDRAÚLIC TURBINES}

English Synopsis p. 119

par M. CUENOD.

Ingénieur E, P. Z.

\section{PLAN DE L'EXPOSÉ}

1. - introduction.

II. - Description de l'installation d'un groupe hydraulique.

III. - Définitions et principes :

a) Fonction de transfert.

b) Courbe de réponse.

c) Courbe de Nyquist.

d) Critère de Nyquist.

IV. - Considérations théoriques permettant de déterminer les caractéristiques dynamiques d'un groupe hydroélectrique :
a) Equation des masses tournantes.
b) Equation de variations de la puis- sance de la turbine.
c) Equation du mouvement de l'eau dans la conduite forcée.
d) Conditions aux limites.

V. - Caractéristique dynamique de la surpression :
a) Fonction de transfert de la sur- pression.
b) Courbe de réponse de la surpres- sion.
1 - Détermination rigoureuse.
2 - Première approximation.
3 - Comparaison avec le résultat obtenu à l'aide de la métho- de d'Alliévi.
4 - Cas particulier d'une conduite à faible chute.
c) Courbe de Nyquist de la surpres- sion. d) Surpression à la suite d'une varia-
tion quelconque de l'ouverture.
e) Conduite à caractéristiques mul- tiples.

VI. - Caractéristique dynamique des variations de puissance de la turbine :
a) Fonction de transfert des varia- tions de puissance de la tur- bine.

b) Courbe de réponse des variations de puissance de la turbine.

c) Courbe de Nyquist des variations de puissance de la turbine.

VII. - Caractéristique dynamique des variations de vitesse :
a) Fonction de transfert des varia- tions de la vitesse.
b) Courbe de réponse des variations de la vitesse.

c) Courbe de Nyquist des variations de la vitesse.

VIII. - Détermination des conditions de stabilité.

IX. - Analogie électrique.

X. - Conclusion. 


\section{I. - INTRODUCTION}

L'étude de la stabilité des turbines hydrauliques a fait l'objet de nombreuses publications (1). Si nous souhaitons y apporter une modeste contribution à l'aide de cet exposé, c'est dans l'idée que l'utilisation de certaines méthodes modernes de calcul, telles que le calcul opérationnel et les transformations de Laplace permettent de traduire les phénomènes qui influent sur la stabilité du réglage de vitesse de sroupes hydroélectriques de façon simple et générale.

L'utilisation du critère de Nyquist auquel ces méthodes conduisent donne la possibilité de tenir compte de certains facteurs que la méthode

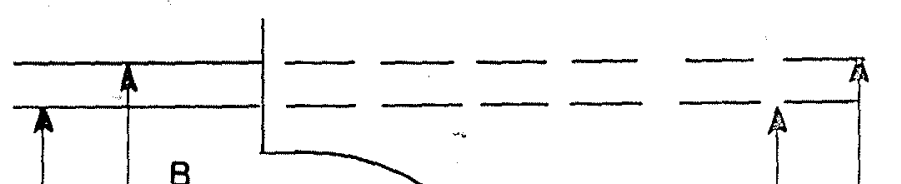

Ainsi que la fig. I en donne le principe, soit la
turbine $T$ entrainant par son couple moteur $M_{t}$
le générateur $G$ qui est branché sur la charge $K$
et quĭ oppose le couple $M_{0}$. Elle est alimentée par

Ainsi que la fig. I en donne le principe, soit la
turbine $T$ entraînant par son couple moteur $M_{t}$
le générateur $G$ qui est branché sur la charge $K$
et quí oppose le couple $M_{0}$. Elle est alimentée par

Ainsi que la fig. I en donne le principe, soit la
turbine $T$ entraînant par son couple moteur $M_{t}$
le générateur $G$ qui est branché sur la charge $K$
et quĭ oppose le couple $M_{0}$. Elle est alimentée par

classique à l'aide du critère de Hurwitz négligeait pour ne pas rendre les calculs trop compliqués.

L'exposé de ces nouveaux modes de calcul et la démonstration du critère de Nyquist a été traité dans plusieurs études $\left({ }^{2}\right)$. Nous nous contentons d'en rappeler rapidement le résultat et $d$ 'indiquer la définition de certaines notions dant nous ferons usage dans la suite de cette étude.

\section{II. - DESCRIPTION DE L'INSTALLATION D'UN GROUPE HYDROELECTRIQUE}

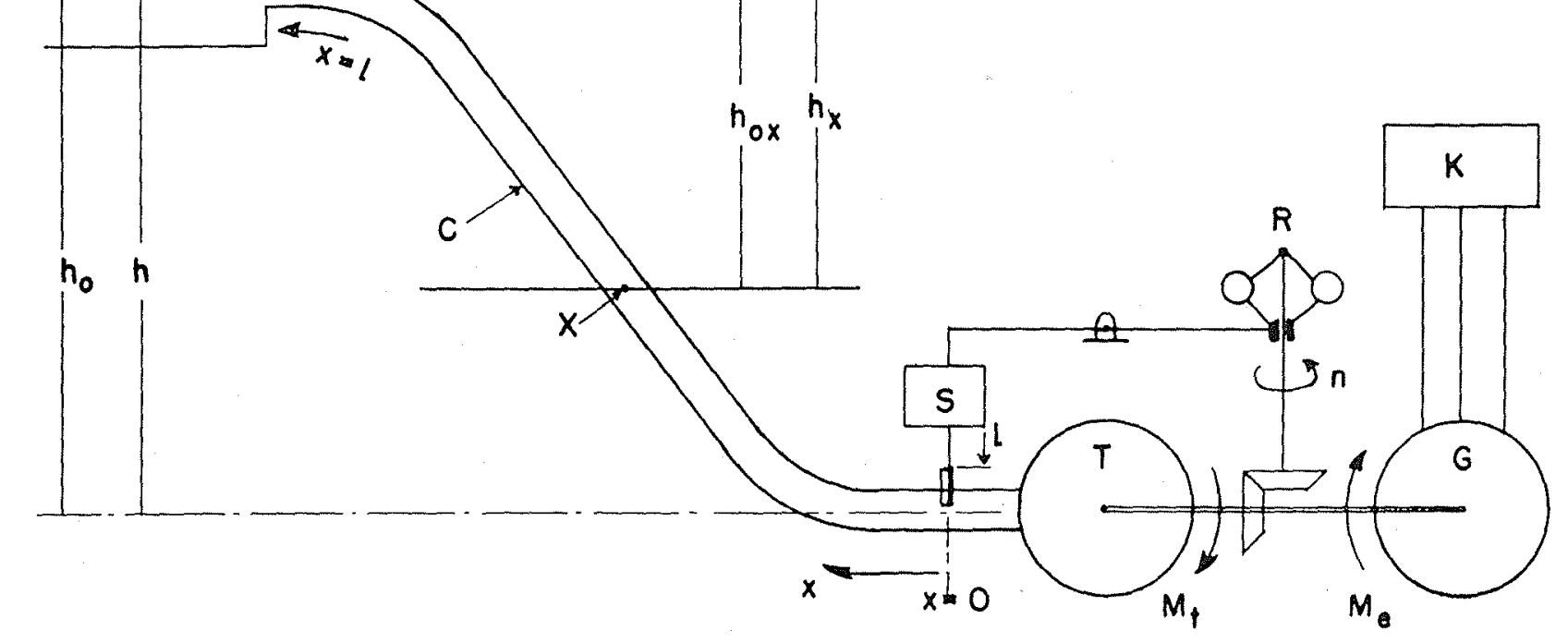

Fig. 1. - Principe de l'installation d'un groupe hydro-électrique.

(1) Voir en particulier:

D. CADEN : Considérations sur le problème de la stabilité. Etude de la stabilité d'un réglage automatique de la vitesse.

M.-P. SATCHE : Théorie des coups de bélier de faible amplitude. Rev. Gén. Electr. Décembre 1947

G. EVANGELISTI: La regolazione delle Turbine idrauliche.

P. ALMERAS : Influence de l'inertie de l'eau sur la stabilité d'un groupe hydroélectrique. "La Houille Blanche $»$, novembre 1945.

J. DANIEL : Accélération du réglage de vitesse des turbines hydrauliques. - "La Houille Blanche», mars-avril 1948.

M. DEJOU : Considérations sur les régulateurs des groupes générateurs hydroélectriques de basse chute. - Rev. Cén. d'Electr. Août 1948.
(2) Voir en particulier:

W. FREY: D'une généralisation du critère de Nyquist. Revue BBC (1946), numéro 3.

Proceedings of the convention on automatic Regulators. The journal of the Institution on electr. Ing. Mai 1947.

OLDENBURG et SARTORIUS: Dynamik der selbsttaegen Regelungen.

M. CUENOD : Etude des propriétés d'un réglage automatique. Bull. Techn. de la Suisse Romande. 26 avril et 10 mai 1947.

C. GALMICHE : Servomécanisme et régulateur. Critères de stabilité, exemple d'application. Rev. Cén. Electr. Janvier 1949.

A. CARDE : The frequency analys method applied to control problem. ASEA Journal, nov. déc. 1948. 
the conduite forcée $C$ de longueur $L$ à partir d'un bassin accumulateur $B$ dont nous admettons le niveau constant, pendant la durée du processus de réglage.

La vitesse de rotation $n$ du groupe est mesurée par le régulateur $R$ qui commande par l'intermédiaire du servo-moteur S I'ouverture I de la turbine.

Nous voyons que ce circuit de réglage peut être décomposé en deux éléments principaux ainsi que le représente schématiquement la fig. 2 :

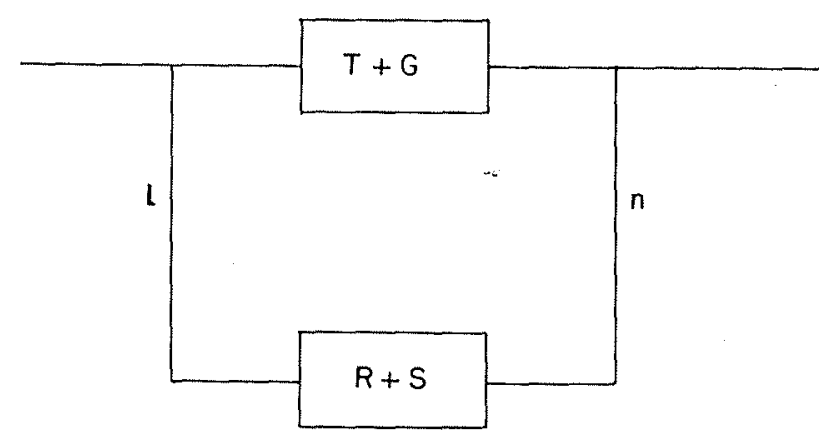

Fig. 2. - Représentation schématique d'un circuit de réglage fermé.

\section{1. - Le groupe turbo-générateur $(T+G)$,} avec:

l'ouverture I comme grandeur d'entrée et la vitesse $n$ comme grandeur de sortie, comprenant la turbine, compte tenu du système hydraulique, le générateur compte tenu de sa charge.

2. - Le dispositif de réglage $(R+S)$, avec: la vitesse $n$ comme grandeur d'entrée et l'ouverture 1 comme grandeur de sortie, comprenant le système d'entraînement du régulateur, le régulateur, les différents amplificateurs et servomoteurs, compte tenu des dispositifs de stabilisation.

Certaines données techniques sont imposées par le genre de l'installation au constructeur, en particulier celles qui concernent le système hydraulique et la charge du générateur ; par contre il est libre dans une certaine mesure, dans le choix du PD* et le choix des caractéristiques dynamiques à donner au dispositif de réglage. Le but de l'étude de la stabilité du réglage de vitesse et partant le but de cet exposé est d'établir certaitaines directives pour ce choix.

\section{III. - DEFINITIONS ET PRINCIPES}

a) Transformation de Loplace. - Depuis l'époque où Heaviside a donné les bases du calcul opérationnel, de nombreuses publications ont été consacrées à cette méthode de calcul (1). Une de ses applications les plus intéressantes concerne l'étude des problèmes de réglage automatique. Nous ne voulons pas en faire un exposé détaillé mais rappeler seulement qu'elle repose sur les transformations de Lap'ace que l'on peut établir à partir de l'intégrale de Fourier.

11 est connu qu'une certaine fonction du temps périodique peut être décomposée selon ses composantes harmoniques:

$$
\begin{aligned}
\Phi(t)=\sum_{-\infty}^{+\infty} c_{\zeta} e^{j \zeta t} \text { avec } c_{k}=\frac{1}{2 \pi} \int_{0}^{2 T} e^{-j k t} \Phi(t) d t \\
j=\sqrt{ }=\sqrt{-1} \\
\zeta=\text { pulsation des oscilla. }
\end{aligned}
$$

tions.

Si la fonction $\Phi(t)$ est apériodique, la période d'intégration doit être étendue de 0 à $\ldots+\infty$ et la somme devient une intégrale avec la pulsation $\zeta$ comme variable d'intégration.

Il en résulte :

$$
\Phi(t)=\int_{-\infty}^{+\infty} e^{j \zeta t} d \zeta \frac{1}{2 \pi} \int_{0}^{-i \zeta t} \Phi(t) d t
$$

Nous multiplions et divisons par $\mathrm{j} \zeta$ et obtenons en posant $\mathrm{j} \zeta=\mathrm{p}$.

$$
\begin{aligned}
& \Phi(t)=\frac{1}{2 \pi j} \int_{-\infty}^{\infty+\infty} \frac{\phi(p)}{p} e^{p t} d p \\
& \phi(p)=p \int_{0}^{\infty} \Phi(t) e^{-p t} d t
\end{aligned}
$$

(1) Voir en particulier :

CARSON : Elektr. Ausgleichvargänge und Operatorenrechnung (1929).

BERG: Das Rechnen mit Operatoren (1932).

DOETSCH : Theorie und Anwendung der Laplace-Transformationen (1937).

DROSTE : Loesung angewandter Dif. Gl. mit LaplaceTransformationen (1937).

Mc. LACHLAN : Complex Variable and Operalcalculation (1939). Modern Operation calculation (1948).

K.-W. WAGNER : Operatorenrechnung (1940).

P. HERRENC : Les applications du calcul opérationnel (1944). 
Le résultat de ces intégrales est rassemblé dans des tables de transformations. Nous indiquons dans le tableau ci-dessous les quelques transformations dont nous nous servons dans cet exposé.

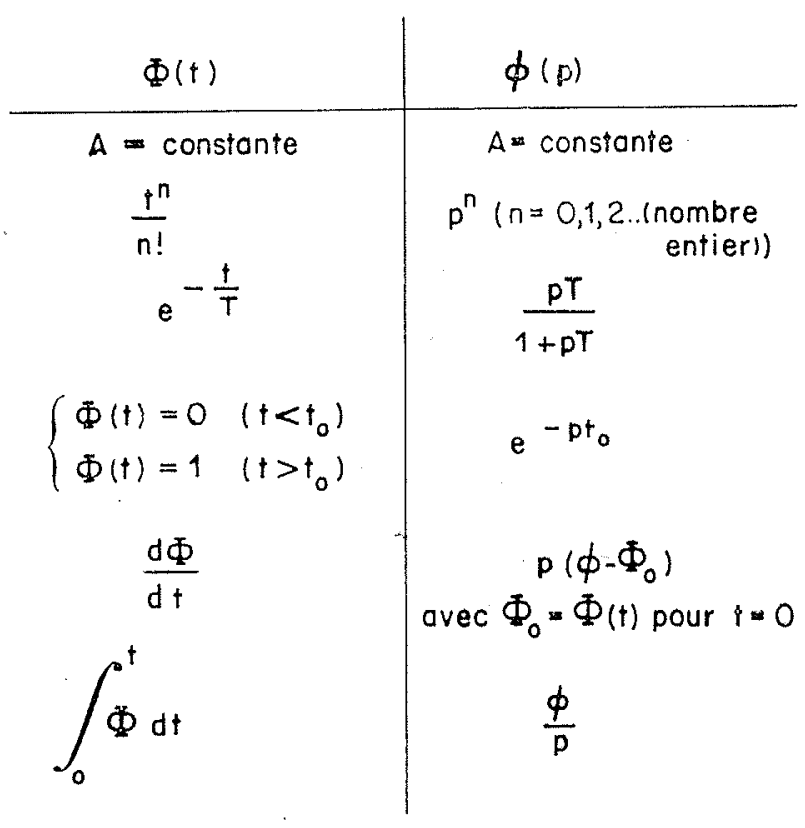

Le calcul opérationnel peut rendre de grands services lorsqu'il s'agit de résoudre un système d'équations différentielles linécires telles que celles que l'on rencontre dans l'étude des problèmes de réglage automatique. Une des propriétés intéressantes de ce mode de calcul est la forme très simple qu'il donne à l'intégrale de Duhamel.

Il est connu, en effet, que lorsque la courbe $A_{1}(t)$ résultant d'une action selon une fonction impulsive unitaire telle que la courbe (a) de la fig. 4 est connue, la courbe $A(t)$ résultant d'une action telle que $A_{2}(t)$ se calcule à l'aide de l'intégrale suivante :

$$
A(t)=\frac{d}{d t} \int_{0}^{t} A_{1}(\xi) A_{2}(t-\xi) d \xi
$$

Cette intégrale sous forme opérationnelle prend la forme très simple suivante: soit a $(p)$, $a_{1}(p), a_{2}(p)$, les transformées de $A, A_{1}, A_{2}$. La fonction a est obtenue tout simplement par le produit de $a_{1}$ et de $a_{2}$.

$$
a=a_{1} \cdot a_{2}
$$

Réciproquement, si la réaction $A(t)$ résultant d'une action $A_{2}(t)$ est connue, il est facile de déterminer la courbe $A_{1}(t)$ qui aurait résulté d'une action selon la fonction unitaire. Sous forme opérationnelle, nous obtenons :

$$
a_{1}=\frac{a}{a_{2}}
$$

C'est cette propriété dont nous faisons usage pour la définition de la fonction de transfert.

b) Relation de transfert. - Nous appelons «relation de transfert» la relation qui relie la grandeur d'entrée à la grandeur de sortie d'un élément du circuit de réglage; cette relation peut être établie théoriquement, ce qui conduit à l'exprimer par une équation différentielle. Elle peut être aussi déterminée expérimentalement :

considérons le dispositif de réglage ouvert tel qu'il est représenté par la fig. 3 :

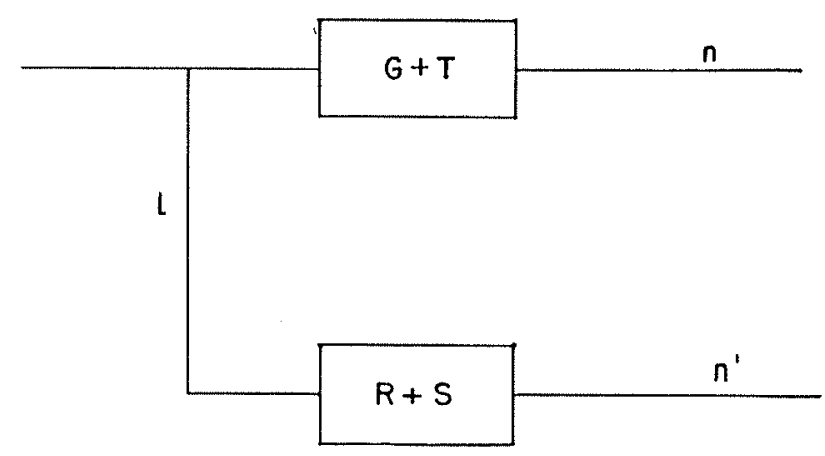

Fig. 3. - Représentation schématique d'un circuit de réglage ouvert.

Nous supposons par exemple que le régulateur $R$ est accouplé à un petit moteur dont nous pouvons faire varier à volonté la vitesse n', indépendamment de la vitesse du groupe $n$.

A partir de la vitesse initiale $n^{\prime}{ }_{0}$ constante, nous la faisons varier selon une fonction $n^{\prime}(t)$ et mesurons la variation de la course I ( $t$ ) à partir de la valeur initiale $I_{0}$.

Nous appelons $\lambda(t)$ et $\nu(t)$ les variations relatives de la vitesse et de la course.

$$
\begin{aligned}
& \nu(t)=\frac{\Delta n^{\prime}}{n_{n}}=\frac{n_{0}^{\prime}-n^{\prime}(t)}{n_{n}} \\
& \lambda(t)=\frac{\Delta l}{l_{\max }}=\frac{l_{0}-l(t)}{l_{\max }}
\end{aligned}
$$

avec $n_{n 1}=$ valeur nominale de la vitesse: avec $\mathrm{I}_{\text {max }}=$ valeur maximale de la course.

La relation de transfert exprime la relation qui existe entre $\nu(t)$ et $\lambda(t)$; elle peut être donnée soit par une équation différentielle avec $\lambda(t)$ comme inconnue, soit sous forme opérationnelle comme le quotient des transformées de Laplace de $\lambda(t)$ et $\nu(t)$, que nous définissons comme étant la fonction de transfert du dispositif de réglage. 


$$
\phi_{\nu \lambda}=\frac{\lambda(p)}{\nu(p)}
$$

b) Courbe de réponse. - Si nous considérons le cas particulier très simple de (t) ayant une allure impulsive, c'est-à-dire si, selon la courbe a de la fig. 4, la vitesse varie brusquement d'une
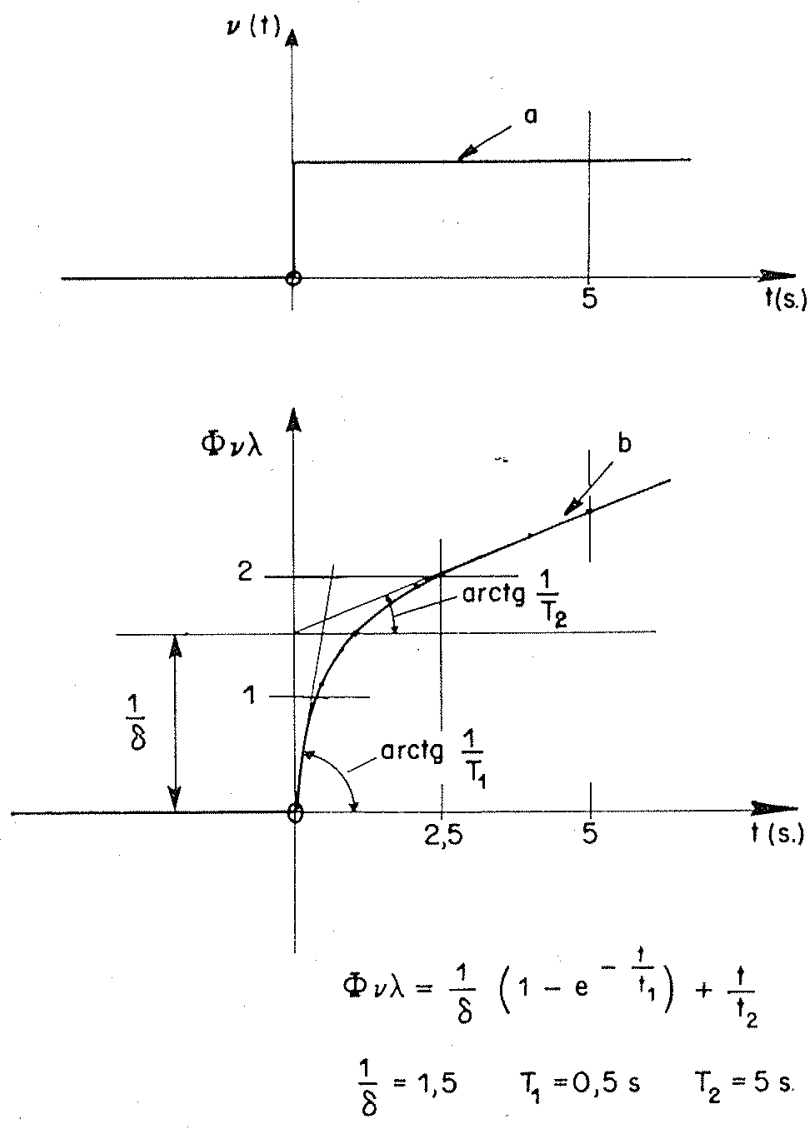

Fig. 4. -...- Courbe de réponse du dispositif de réglage

certaine quantité et reste ensuite constante, la variation relative de la course $\Phi_{y, j}(t)$ qui en résulte est définie comme la courbe de réponse.

La courbe b de la fig. 4 représente la courbe de réponse d'un dispositif de réglage astatique avec statisme passager; de cette courbe que nous supposons relevée expérimentalement, nous déduisons l'expression mathématique :

$$
\Phi_{\nu \lambda}=\frac{\lambda(1)}{\nu}=\frac{1}{\delta}\left(1-e^{-\frac{1}{T_{1}}}\right)+\frac{1}{T_{2}}
$$

avec $\delta=$ statisme passager $(30 \div 150 \%)$. $T_{1}=$ temps de réaction $=$ temps que met le servo-moteur à parcourir a \% de sa course après un écart de vitesse a \% pendant la première partie de son action avant que l'amortissement n'intervienne $\left(T_{1}=0,1 \div 0,8\right.$ sec.).
$T_{9}=$ temps d'amortissement $=$ temps que met le servo-moteur pour parcourir a $\%$ de sa course après un écart de vitesse de c \% la vitesse étant constante :

$$
\left(T_{2}=1 \div 10 \mathrm{sec} .\right)
$$

Nous obtenons la fonction de transfert en appliquant la transformation de Laplace à l'expression mathématique de la courbe de réponse $\Phi$.

$$
\Phi_{\nu \lambda} \frac{1}{\delta} \frac{1}{p T_{1}+1}+\frac{1}{p T_{2}}
$$

11 est bien évident que si la relation de transfert a été établie théoriquement à l'aide d'équation différentielle, il est très aisé d'en déterminer l'expression de la fonction de transfert qui, comme nous le verrons, offre de grands avantages. La courbe de réponse peut alors se calculer en appliquant la transformation de Laplace à la fonction de transfert.

c) Courbe de Nyquist. - Les caractéristiques dynamiques d'un organe du circuit de réglage peuvent être enregistrées par un autre essai. Nous faisons varier la grandeur d'entrée selon une course sinusoïdale de faible amplitude et de pulsation $\zeta$ autour d'une valeur moyenne. La grandeur de sortie oscillera également autour. d'une valeur moyenne selon une oscillation har. monique dont nous mesurons l'amplitude et le déphasage par rapport à l'oscillation de la grandeur d'entrée. Nous exprimons le résultat de cette mesure par un vecteur $J\left(\zeta_{1}\right)$ représenté par la figure 5 dont la grandeur absolue est

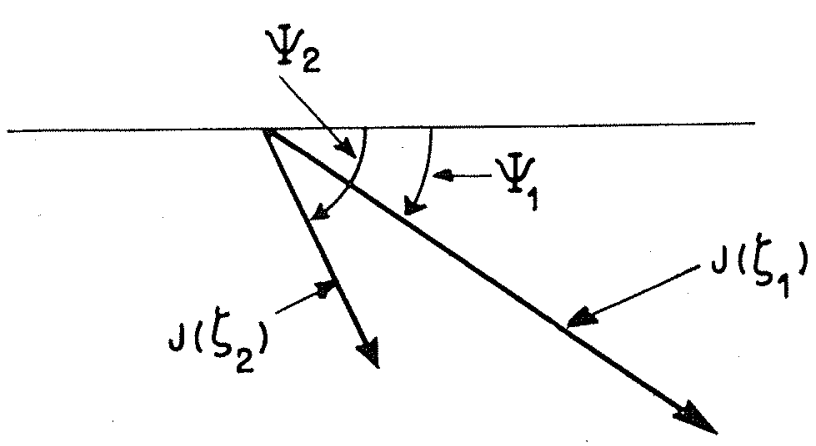

Fig. 5. - Détermination de la courbe de Nyquist.

égale au rapport de l'amplitude des deux oscillations et la phase $\psi_{\mathrm{l}}$, égale au déphasage entre ces deux oscillations.

Si nous répétons le même essai avec une oscillation ayant d'autres pulsations $\zeta_{2} \zeta_{3}$ nous obtiendrons des vecteurs $J\left(\zeta_{2}\right), J\left(\zeta_{3}\right)$ ayant en règle générale différentes phases et grandeurs absolues. Si nous joignons l'extrémité de ces différents vecteurs nous obtenons une courbe cotée 
en fonction de $\zeta$ que nous appelons la courbe de Nyquist.

Au lieu d'être relevée expérimentalement, ce qui nécessiterait souvent des essais compliqués,

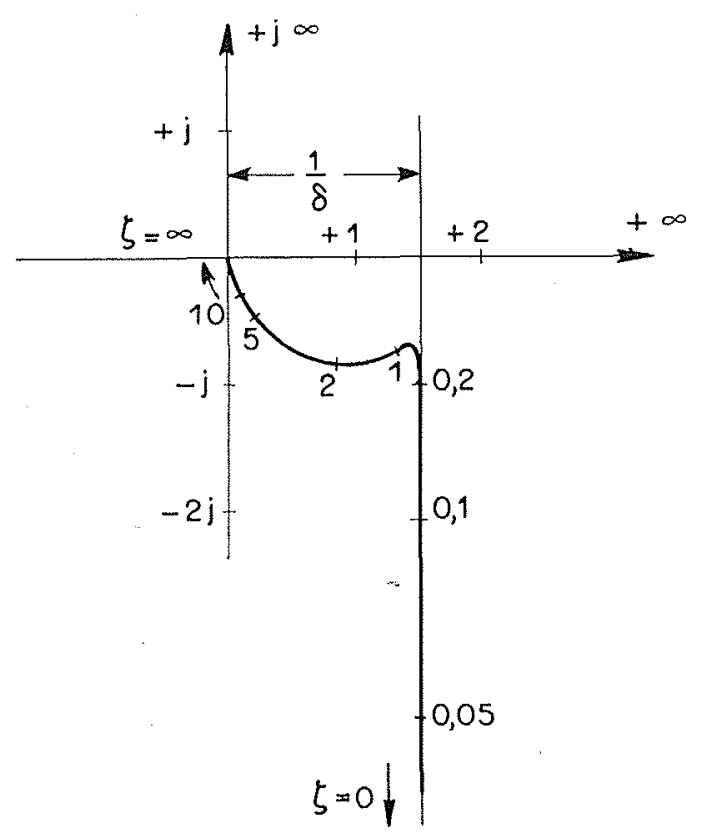

$$
J_{\nu \lambda}=\frac{1}{\delta} \frac{1}{j \zeta T_{1}+1}+\frac{1}{j \zeta T_{2}} \quad \frac{1}{\delta}=1,5 \quad T_{1}=0,5 s \quad T_{2}=5 s
$$

Fig. 6. - Courbe de Nyquist du dispositif de réglage sous forme vectorielle.

cette courbe de Nyquist peut être calculée à partir de l'équation différentielle du dispositif de

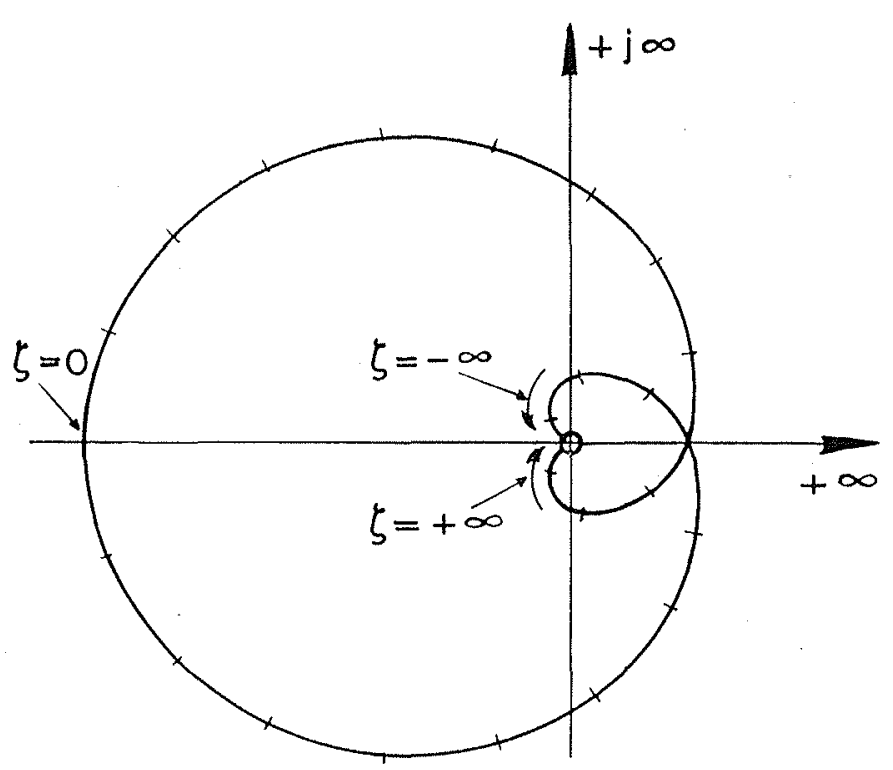

réglage ou déduite de la courbe de réponse ; il suffit de remplacer l'opérateur p par $j \zeta$ dans la fonction de transfert pour avoir dans le plan complexe l'équation de la courbe de Nyquist du dispositif de réglage dont nous connaissons la fonction de transfert :

La fig. 6 représente la courbe de Nyquist sous forme vectorielle d'un dispositif de réglage astatique avec statisme passager avec les valeurs numériques correspondant à celles pour lesquelles la courbe de réponse de la fig. 4 a été dessinée. Les courbes $J \nu \lambda$ et $\psi \nu \lambda$ de la fig. 21 donnent une représentation cartésienne de cette courbe de Nyquist.

d) Critère de Nyquist. - Lorsque les courbes de Nyquist de chaque élément du circuit de réglage ont été établies, la courbe de Nyquist du circuit de réglage s'obtient en faisant le produit des différentes courbes de Nyquist (c'est-à-dire pour chaque pulsation en additionnant leur phase et en faisant le produit de leur grandeur absolue) :

$$
J_{R}=-J_{\nu \lambda} \cdot J_{\lambda \nu}
$$

Le signe négatif signifie que le dispositif de réglage agit de façon à corriger l'écart donc en sens inverse de l'écart initial de la grandeur à régler.

Si nous dessinons la courbe symétrique par rapport à l'axe réel, nous obtenons la courbe de Nyquist pour des valeurs de $\zeta$ négatives.

Ainsi qu'on peut le démontrer ("), le réglage est

(1) FREY : D'une généralisation du critère de Nyquist Revue BBC - 1946, n० 3.

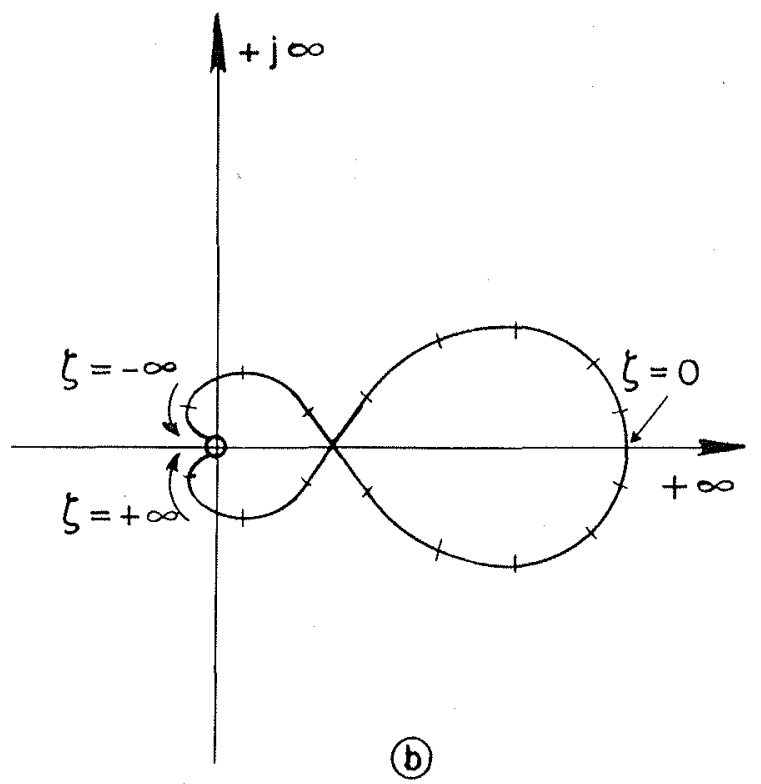

(a) Fig. 7. - Critère de Nyquist.

a) Circuit de réglage ouvert stable.

b) Circuit de réglage ouvert instable. 
stable si, lorsqu'on parcourt lo courbe de Nyquist du réglage de $\zeta=+\infty$ à $\zeta=-\infty$ on entoure le point $(+1$, jo) dans le sens des aiguilles d'une montre autant de fois que le système ouvert a de racines habiles.

Ainsi, si le système ouvert est stable (voir fig. 7a) le point $(-1$, jo) ne doit pas être entouré par la courbe de Nyquist. Si le système ouvert comporte une instabilité du premier ordre (voir fig. 7D) le point $(+1, j o)$ doit être entouré une fois.

La démonstration du critère de Nyquist relève de la théorie des fonctions. Nous nous proposons seulement d'en rendre plausible la signification physique à l'aide des considérations suivantes: soit un circuit de réglage ouvert stable ayant une courbe de Nyquist telle que celle qui est représentée par la fig. 7a. Pour une certaine fréquence, les oscillations à la «sortie» du dispositif de réglage seront en phase avec celtes à l' «entrée » du dispositif. Si elles sont de même amplitude, rien ne serait changé si on "fermait " le circuit de réglage, les oscillations continueraient à amplitude constante. Ce cas représente le cas limite de stabilité. Si, au contraire, les oscil!ations de sortie sont plus faibles que les oscillations d'entrée, on se rend compte aisément que les oscillations de réglage s'amortiraient d'elles-mêmes si on fermait le circuit de réglage, tandis que, au contraire, elles iraient en s'amplifiant si les oscillations de sortie étaient plus élevées que les oscillations d'entrée. On comprend que la position de la courbe de Nyquist par rapport au point $(+1$, jo) qui représente l'extrémité du vecteur de l'oscillation d'entrée du dispositif de réglage permette de tirer des conclusions immédiates sur sa stabilité.

\section{IV. - CONSIDERATIONS THEORIQUES PERMETTANT DE DETERMINER LES CARACTERISTIQUES DYNAMIQUES D'UN GROUPE HYDROELECTRIQUE.}

a) Equation des masses tournantes. - L'équation différentielle écrite en valeur relative décrivant les variations de vitesse d'un groupe électrogène est classique :

$$
T_{a} \frac{d \frac{n}{n_{n}}}{d t}+\frac{M_{e}}{M_{\theta_{n}}}=\frac{M_{t}}{M_{t}}
$$

avec $M_{t}=$ couple moteur exercé par la turbine.

$M_{e}=$ couple résistant électrique.

$$
\begin{aligned}
& T_{\mathrm{a}}=\text { constante d'accélération. } \\
& T_{\mathrm{a}}=\mathrm{\omega}_{\mathrm{a}} \\
& \mathrm{M}_{\mathrm{a}}
\end{aligned}
$$$$
\Theta=\text { moment d'inertie polaire des mas- }
$$
ses tournantes.

$$
\omega_{1}=\text { vitesse angulaire } \frac{2 \pi \mathrm{n}_{\mathrm{n}}}{60}
$$

$M r_{n}, M z_{n}, n_{n}=$ valeur de consigne de $M_{1} M_{n} n$.

Nous considérons à nouveau de faibles variations par rapport à un certain état initial caractérisé par les grandeurs $n_{0}, M z_{0}$ et $M t_{n}$

Nous posons :

$$
\begin{aligned}
& n=n o+\Delta n \\
& \mu_{e}=\frac{\Delta M_{e}}{M_{e_{n}}} \\
& M_{t}=M_{\dagger_{0}}+\Delta M_{\dagger} \\
& M_{0}=M_{0_{0}}+\Delta M_{0} \\
& \mu_{t}=\frac{\Delta M_{t}}{M_{t_{n}}}
\end{aligned}
$$

et $\nu=\frac{\Delta_{1}}{n_{n}}$ variation relative de la charge.

La variation du couple moteur dépend des vaviations de l'ouverture et des variations de la vitesse :

$$
\mu_{\uparrow}=\frac{\delta \frac{M_{0}}{M_{n}}}{\delta \frac{L}{l_{n}}} \lambda+\frac{\delta \frac{M_{t}}{M_{n}}}{\delta \frac{n}{n_{n}}} \nu=\phi_{\lambda \mu_{t}} \lambda+\phi_{\nu \mu_{t}} \nu
$$

Les variations du couple résistant dépendent des variations de la charge $K$ et des variations de la vitesse :

$$
\mu_{e}=\frac{\delta \frac{M_{e}}{M_{n}}}{\delta \frac{k}{K_{n}}} \cdot \kappa+\frac{\delta \frac{M_{e}}{M_{n}}}{\delta \frac{n}{n_{n}}} \cdot \nu=\phi_{K \mu_{e}} \cdot \kappa+\phi_{\nu \mu_{e}} \cdot \nu
$$

cvec $k=$ variations relative de la charge.

$\phi_{\lambda_{\mu_{t}}}=$ fonction de transfert de la puissance de la turbine par rapport

$$
\phi_{\nu \mu_{t}}=\begin{gathered}
\text { aux variations d'ouverture. } \\
\text { fonction de transfert du couple }
\end{gathered}
$$
moteur par rapport aux varia-

$\phi_{k \mu_{e}}=\begin{gathered}\text { tions de vitesse. } \\ \text { fonction de transfert de la puis. }\end{gathered}$ sance consommée par rapport

$\phi \nu \mu_{\mathrm{e}}=\begin{gathered}\text { aux variations de la charge. } \\ \text { fonction de transfert du couple }\end{gathered}$ résistant par rapport aux variations de vitesse.

Selon la fig. 8, le diagramme des couples moteurs en fonction de la vitesse est représenté par une famille de courbes $T_{0}, T_{1}, T_{2}$, correspondant en régime permanent aux différentes ouvertures 
de la turbine. Le diagramme des couples résistants est représenté par une famille de courbes $G_{0}, G_{1}$, $G_{2}$ correspondant aux différentes charges. Ce diagramme permet de déterminer pour une vitesse $n_{0}$ et une charge correspondant à la courbe $G_{0}$ l'ouverture nécessaire correspondant à la courbe $T_{0}$ coupant $G_{0}$ à l'abscisse $n_{0}$.

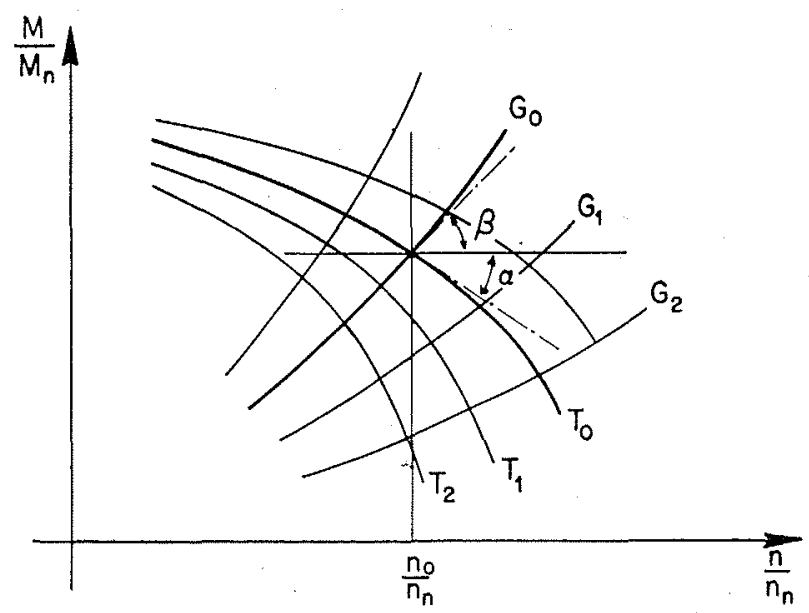

Fig. 8. - Détermination des variations relatives des couples moteurs et résistants en fonction des variotions de vitesse.

En négligeant les phénomènes transitoires du mouvement de l'eau dans la turbine et de l'excitation du générateur, ce qui est légitime parce que la constante de temps du processus de réglage est beaucoup plus grande que celle des phénomènes transitoires de la turbine et du générateur, si nous partons d'un état initial $T_{0}$ et $G_{0}$, nous pouvons déduire en première approximation les fonctions de transfert en assimilant les caractéristiques des couples aux environs du point de marche à leur tangente en ce point.

$$
\begin{aligned}
& \phi_{\nu \mu_{t}}=\operatorname{tg} \alpha \\
& \phi_{\nu \mu_{e}}=\operatorname{tg} \beta
\end{aligned}
$$

En tenant compte que jusqu'à la période initiale la vitesse étant constante, $c^{\prime}$ est-à-dire que $\frac{M_{\text {eO }}}{M_{\text {en }}}=\frac{M_{10}}{M_{\text {tn }}}$

et en introduisant les valeurs

relatives des variations, l'équation différentielle des masses tournantes prend la forme suivante, écrite sous forme opérationnelle :

$$
\mathrm{T}_{a} \cdot p \cdot \nu+\phi_{\kappa \mu_{0}} k+\phi_{\nu \mu_{e}}^{\nu=} \phi_{\lambda \mu_{t}} \lambda+\phi_{\nu \mu_{t}} \cdot \nu
$$

Nous supposons la charge constante $(x=0)$ et nous introduisons les valeurs des fonctions de transfert des couples en fonction de la vitesse que nous déduisons de la fig. 8 . II résulte:

$$
T_{a} \cdot p \cdot \nu+\nu(\operatorname{tg} \beta-\operatorname{tg} \alpha)=\phi_{\lambda \mu_{t}} \lambda
$$

D'où nous déduisons la forction de transfert des variations de vitesse par rapport aux variations de l'ouverture :

$$
\phi_{\lambda \nu}=\frac{\nu}{\lambda}=\frac{\phi_{\lambda \mu_{1}}}{p T_{a}+\operatorname{tg} \beta-\operatorname{tg} \alpha}
$$

Nous admettons comme cas particulier que la puissance de la turbine et du générateur est constante indépendamment de la vitesse, ce qui suppose que le réglage à tension constante est infiniment rapide avec charge purement ohmique et que la turbine marche à un régime correspondant au sommet de sa caractéristique ( $\left.{ }^{1}\right)$. Nous obtenons :

$$
\begin{aligned}
& \frac{M}{M_{n}}=\frac{P}{P_{n}} \frac{n_{n}}{n} \\
& \frac{\delta \frac{M}{M_{n}}}{\delta \frac{n}{n_{n}}}=-\frac{P}{P_{n}}\left(\frac{n_{n}}{n}\right)^{2}
\end{aligned}
$$

$\mathrm{Si}$ nous admettons qu'à l'état initial, nous avons les valeurs nominales, il résulte:

$$
\phi_{\nu \mu_{t}}=\phi_{\nu \mu_{e}}=\operatorname{tg} \alpha=\operatorname{tg} \beta=-1
$$

l'équation différentielle des masses tournantes prend la forme très simple suivante :

$$
T_{a} p \nu=\phi_{\lambda \mu_{t}} \lambda
$$

et la fonction de transfert des variations de vitesse :

$$
\phi_{\lambda \nu}=\frac{\phi_{\lambda \mu_{t}}}{p T_{a}}
$$

b) Equation des variations de puissance de la turbine. - La fonction de transfert du couple moteur par rapport aux variations de l'ouverture doit être établie en supposant que la vitesse est constante. Dans ce cas, la valeur relative du couple moteur est égale à la valeur relative de la puissance de la turbine c'est-à-dire au produit de la valeur relative du débit par la valeur relative de la pression. Nous obtenons :

$$
\frac{M_{1}}{M_{t_{n}}}=\frac{1}{l_{\max }}\left(\frac{H}{H_{0}}\right)^{\frac{3}{2}}
$$

(1) C'est-à-dire que par rapport aux variations de charge, le rendement est constant. 
avec $\frac{\mathrm{H}}{\mathrm{H}_{0}}=(1+\sigma)=$ pression relative de la colonne d'eau.

$$
\sigma=\text { surpression relative. }
$$

Nous considérons à nouveau de petites variations à partir d'un certain état initial $M_{0}, I_{0}$ $\mathrm{H}_{0}$.

La valeur relative initiale de l'ouverture de la turbine nous donne la charge relative initiale de la turbine $\lambda_{0}$ :

$$
\lambda_{0}=\frac{L_{0}}{L_{\text {max }}}=\frac{M_{t_{0}}}{M_{t_{n}}}
$$

nous obtenons en première approximation :

$$
\mu_{t}=\lambda+\frac{3}{2} \lambda_{0} \sigma
$$

En admettant que les variations du rendement de la turbine soient négligeables, nous voyons que le couple moteur dépend de l'ouverture de la turbine et de la surpression, celte-ci dépendant elle aussi des variations de l'ouverture. Il en sésulte :

$$
\mu_{t}=\lambda+\frac{3}{2} \lambda_{0} \lambda \phi_{\lambda \sigma}
$$

avec $\varphi \lambda_{\sigma}=$ fonction de transfert de la surpression.

Nous en déduisons l'expression de la fonction de transfert des variations de puissance:

$$
\phi_{\lambda \mu_{t}}=\frac{\mu_{t}}{\lambda}=1+\frac{3}{2} \lambda_{0} \phi_{\lambda \sigma}
$$

c) Equation du mouvement de l'eau dans une conduite forcée. - Pour simplifier les calculs, nous supposons que la conduite est à caractéristique unique, et nous négligeons les pertes dues au frottement, à la contraction à l'entrée de la conduite, ainsi que la hauteur figurative de l'énergie cinétique.

Le système d'équation différentielle à dérivée partielle qui décrit le mouvement non permanent de l'eau dans les conduites est classique:

$$
\left\{\begin{array}{l}
\frac{\delta v}{\delta t}=g \frac{\delta H}{\delta x} \\
\frac{\delta v}{\delta x}=\frac{g}{a^{2}} \frac{\delta H}{\delta t}
\end{array}\right.
$$

$v=$ vitesse de l'eau.

$a=$ vitesse de propagation des ondes de pression.

$$
a=\sqrt{\frac{\frac{g}{Y_{w}}}{\epsilon}+\frac{D}{E e}}
$$

avec $g=$ constante d'accélération.

$\gamma_{w}=$ poids spécifique de l'eau.

$\varepsilon=$ coefficient de compressibilité de l'eau.

$\mathrm{D}=$ diamètre de la conduite.

$E=$ module d'élasticité des parois.

e = épaisseur des parois.

Exemple : pour $\varepsilon=2,07 \quad 1 \mathrm{C}^{4} \mathrm{~kg} / \mathrm{cm}^{2}$ à la température de $15^{\circ}$.

$$
\begin{aligned}
& g=981 \mathrm{~cm} / \mathrm{sec}^{2} \\
& y_{\mathrm{E}}=0,001 \mathrm{~kg} / \mathrm{cm}^{3} \\
& \frac{D}{e}=100(\mathrm{D}=1 \mathrm{~m}, e=1 \mathrm{~cm})
\end{aligned}
$$

nous obtenons :

$$
a=\sqrt{\frac{\frac{981}{0,001}}{\frac{1}{2,07.10^{4}}+\frac{100}{2,15.10^{6}}}}=1017^{\mathrm{m} / \mathrm{sec}}
$$

En éliminant l'une ou l'autre des variables, nous obtenons les deux équations linéaires suivantes:

$$
\frac{\delta^{2} v}{\delta x^{2}}=\frac{1}{a^{2}} \frac{\delta^{2} v}{\delta t^{2}}, \quad \frac{\delta^{2} H}{\delta x^{2}}=\frac{1}{a^{2}} \frac{\delta^{2} H}{\delta t^{2}}
$$

Leur solution générale est connue :

$$
\begin{aligned}
& H=H_{0}+F\left(t-\frac{x}{a}\right)+f\left(t+\frac{x}{a}\right) \\
& v=v_{0}-\frac{\dot{g}}{a}\left(F\left(t-\frac{x}{a}\right)-f\left(t+\frac{x}{a}\right)\right)
\end{aligned}
$$

$F$ et $f$ sont des fonctions qu'il faut déterminer en tenant compte des conditions aux limites. Elles représentent deux systèmes d'onde qui be propagent sans se déformer avec la vitesse a en sens inverse l'une de l'autre, la vitesse $v$ 'étant négligeable par rapport à la vitesse a. La pression en un point quelconque de la conduite est don. née par la superposition de ces deux fonctions. Sa détermination peut se faire soit analytiquement par la méthode d'Alliévi, soit graphiquement par la méthode de Schnyder-Bergeron. Nous allons indiquer une troisième méthode qui, sans rien apporter en principe de nouveau, fait apparaitre le résultat sous une forme commode pour l'étude de la stabilité du réglage de vitesse.

Nous appliquons la transformation de Laplace aux équations différentielles décrivant le mouvement de l'eau dans la conduite forcée :

$$
\frac{\delta^{2} v}{\delta x^{2}}=\frac{p^{2}}{a^{2}} v, \frac{\delta^{2} H}{\delta x^{2}}=\frac{p^{2}}{a^{2}} H
$$

Résolues par rapport à $x$, elles conduisent aux solutions suivantes: 


$$
\left\{\begin{array}{l}
H_{x}=H_{0}+F e^{-\frac{p}{a} x}+f e^{+\frac{p}{a} x} \\
v_{x}=v_{0}-\frac{g}{a}\left(F e^{-\frac{p}{a} x}-f e^{-\frac{p}{a} x}\right)
\end{array}\right.
$$

Ecrites sous une forme un peu différente, et en tenant compte des valeurs $H_{e}$ et $v_{e}$ en amont de la conduite nous obtenons, sous forme opérationnelle la pression $H_{x}$ et la vitesse $v_{x}$ en un point quelconque $d^{\prime a b s c i s s e ~} x$ de la conduite.

$$
\left\{\begin{array}{l}
H_{x}=H_{o_{x}}+H_{e} \operatorname{ch} p\left(\frac{L-x}{a}\right)+\frac{a}{g} \operatorname{shp}\left(\frac{L-x}{a}\right) \\
v_{x}=v_{o_{x}}+H_{e} \frac{g}{a} \operatorname{shp}\left(\frac{L-x}{a}\right)+v_{e} \operatorname{ch} p\left(\frac{L-x}{a}\right)
\end{array}\right.
$$

en particulier au début de la conduite $(x=0)$ nous obtenons, en ne considérant de nouveau que les variations relatives de la pression $\sigma$ et de la vitesse $y$

$$
\begin{aligned}
& \sigma=\sigma_{e} \operatorname{chp} \frac{T_{r}}{2}+2 \rho \gamma_{e} \operatorname{shp} \frac{T_{r}}{2} \\
& \gamma=\frac{\sigma_{e}}{2 \rho} \operatorname{shp} \frac{T_{r}}{2}+\gamma \operatorname{chp} \frac{T_{r}}{2} \\
& \text { ovec } \rho=\frac{a}{2 g} \frac{v_{\max }}{H_{0}}=
\end{aligned}
$$

chiffre caractéristique de la conduite.

$$
\text { - } \sigma_{0}, \gamma_{0}
$$

variation relative de la pression et de la vitess? à la fin de la conduite (côté amont).

$v_{\max }=$ vitesse de l'eau dans la conduite à pleine ouverture.

variation relative de la pres-

$\sigma, \gamma=$ sion et de la vitesse au début de la conduite (côté aval). $T_{r}=\frac{1}{2 f_{r}}=\frac{2 L}{a}=\begin{gathered}\text { demi-période de résonance de } \\ \text { la conduite. }\end{gathered}$ $f_{r}=$ fréquence de résonance de la conduite.

d) Etablissement des conditions aux limites.

1) Conditions au début de la conduite.

La vitesse à la sortie de l'obturateur est proportionnelle à l'ouverture et à la racine carrée de la pression ; en valeur relative, nous obtenons :

$$
-\frac{v}{v_{\max }}=\sqrt{\frac{H}{H_{0}}} \cdot \frac{l}{l_{\max }}
$$

le signe négatif du terme de gauche s'explique par le fait que les $x$ étant comptés en remontant la conduite, la vitesse doit être considérée comme négative; en ne considérant de nouveau que de faibles variations relatives, nous obtenons en première opproximation :

$$
-\gamma=\frac{\lambda_{0}}{2} \sigma+\lambda
$$

\section{2) Conditions à la fin de la conduite.}

Nous avons admis que le niveau du bassin d'accumulation ne varie pratiquement pas pendant la durée de la manœuvre de lobturateur, donc la pression $H_{0}$ peut être considérée comme constante; il en résulte en première approximation :

$$
\sigma_{0}=0
$$

\section{V. - CARACTERISTIQUE DYNAMIQUE DE LA SURPRESSION.}

a) Fonction de transfert de la surpression. A l'aide des conditions aux limites, nous élimi. nons la variation relative de la vitesse et nous déterminons la fonction de transfert de la sur. pression en un point quelconque de la conduite résultant d'une variation relative $\lambda$ de l'ouverture de la turbine:

$$
\phi_{\lambda \sigma_{x}}=\frac{\sigma_{x}}{\lambda}=-\frac{2 \rho \operatorname{sh} p\left(\frac{L-x}{a}\right)}{\operatorname{ch} p \frac{T_{r}}{2}+\rho \lambda_{0} \operatorname{sh} p \frac{T_{r}}{2}}
$$

le signe négatif du terme de droite signifie qu'à la suite d'une ouverture $(\lambda>0)$ il résulte une dépression $(\sigma<0)$.

En particulier, au début de la conduite, nous obtenons :

$$
\phi_{\lambda \sigma}=\frac{\sigma}{\lambda}=-\frac{2 \rho}{\rho \lambda_{0}+\operatorname{coth} p \frac{T_{r}}{2}}
$$

b) Courbe de réponse de la surpression. Nous déterminons les variations de pression en aval de la conduite résultant d'une petite varıation supposée infiniment rapide de l'ouverture.

1. - Détermination rigoureuse. - Nous appliquons la formule de Heaviside à la fonction de transfert :

$$
\Phi_{\lambda \sigma}=\frac{\sigma(t)}{\lambda}=-\sum_{p 1 \ldots p n} 4 \rho \frac{e^{p t} s h^{2} p \frac{T_{r}}{2}}{p T_{r}}
$$

$P_{1}, P_{n}=$ racine de l'équation. $p \lambda_{0}+\operatorname{coth} p \frac{T r}{2}=0$

Cette équation est transcendante, elle peut être aisément résolue graphiquement dans un cas 
particulier, mais se prête mal à une discussion générale, aussi dans la suite nous contenteronsnous du résultat que donne une première approximátion à l'aide d'un développement en série.

2. - Première approximation. - Nous remplaçons coth par son expression sous forme exponentielle:

$$
\begin{aligned}
& { }_{\lambda \sigma}=-\frac{2 \rho}{e^{+p \frac{T_{r}}{2}}+e^{-p \frac{T_{r}}{2}}}=\frac{2 \rho}{\rho \lambda_{0}+1} \frac{1-e^{-p T_{r}}}{1-r e^{-p T_{r}}} \\
& \rho \lambda_{0}+\frac{e^{+p \frac{T_{r}}{2}}+e^{-p \frac{T_{r}}{2}}}{e^{+p \frac{T_{r}}{2}}-e^{-p \frac{T_{r}}{2}}}
\end{aligned}
$$

avec $r=\frac{\lambda_{0} \rho-1}{\lambda_{0} \rho+1}=$ coefficient de réflexion.

Comme $|r|$ est $<1$ nous pouvons développer

$$
\frac{1}{1-r e^{-p T_{r}}}
$$

$$
\frac{1}{1-r e^{-p T_{r}}}=1+r e^{-p T_{r}}+r^{2} e^{-2 p T_{r}}+\ldots
$$

Nous tenons compte du fait que

$\frac{1-e^{-p T_{r}}}{1-r e^{-p T_{r}}}=1-(1-r)\left(e^{-p T_{r}}+r e^{-2 p T_{r}}+r^{2} e^{-3 p T_{r}}+.\right)$ et que

$$
1-r=1+\frac{1-\rho \lambda_{0}}{1+\rho \lambda_{0}}=\frac{2}{1+p \lambda_{0}}
$$

Il en résulte la fonction de transfert suivante:

$$
\begin{array}{r}
\phi_{\lambda \sigma}=-\rho(1-r)\left(1-(1-r)\left(e^{-p T_{r}}+r e^{-2 p T_{r}}\right.\right. \\
+r^{2} e^{-3 p T_{r}}+. . r^{n} e^{\left.\left.-(n+1) p T_{r}\right)\right)}
\end{array}
$$

Le produit de la fonction $\varphi(p)$ avec la fonc tion opérationnelle e-pTr dans le domaine opéra teur correspond dans le domaine temps à un décalage du laps de temps $\mathrm{Tr}$; nous voyons immédiatement que la courbe de réponse $\Phi_{\lambda \sigma}$ apparaît comme une superposition de fonctions impulsives intervenant successivement après les laps de temps $T_{r}, 2 T_{r}, 3 T_{r}, \ldots . .11$ en résulte comme courbe de réponse, une courbe en escalier telle que (fig. 10) :

$$
\begin{array}{cc}
0<t<T_{r} & \Phi_{\lambda \sigma}=-\rho(1-r) \\
T_{r}<t<2 T_{r} & \Phi_{\lambda \sigma}=-\rho(1-r) r \\
2 T_{r}<t<3 T_{r} & \Phi_{\lambda \sigma}=-\rho(1-r) r^{2} \\
\vdots & \vdots \\
n T_{r}<t<(n+1) T_{r} & \Phi_{\lambda \sigma}=-\rho(1-r) r^{n}
\end{array}
$$

Comme $r<1$ il résulte que $r^{n} \rightarrow 0$ si $n=\infty$ : la surpression s'annule au bout d'un certain temps.

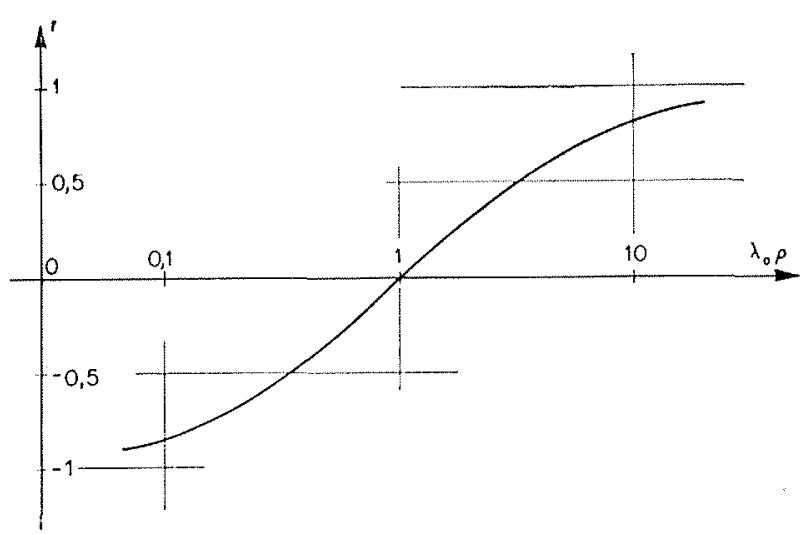

Fig. 9. - Détermination du facteur de réflexion.

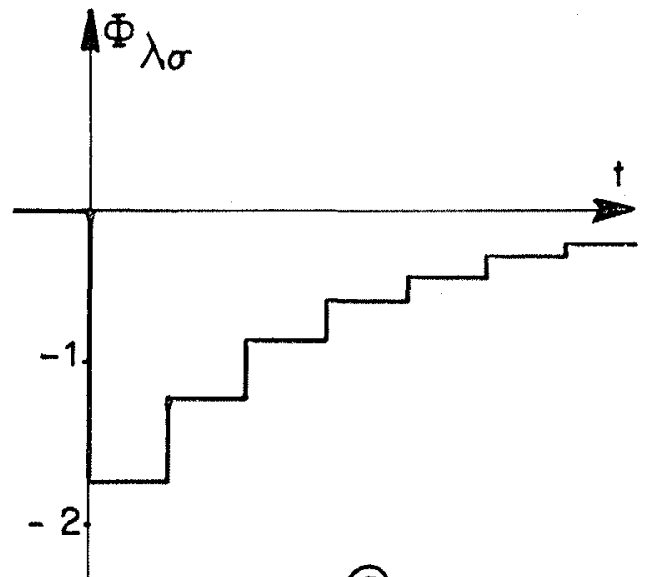

(a)

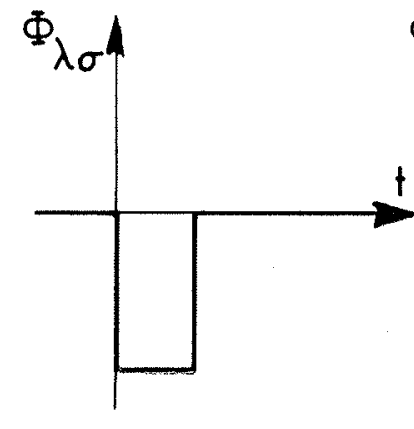

(b)

(C)

Fig. :0. - Courbe de réponse de la surpression 
La valeur du facteur de réflexion qui peut se déduire de la fig. 9 caractérise cette courbe de réponse. Nous pouvons distinguer trois cas principaux :

$$
\begin{array}{lcc}
\rho \lambda_{0}>1 \quad r>0 & & \rho \lambda_{0}<1 \quad r<0 \\
\lambda_{0}=1 & \lambda_{0}=1 & \lambda_{0}=1 \\
\rho=5,7 \quad r=0,7 & \rho=1 \quad r=0 & \rho=0,38 \quad r=-0,4
\end{array}
$$

a)

b)

c)

$$
P_{\lambda_{0}}>1 \quad 1>r>0
$$

C'est le cas d'une conduite à faible hauteur de chute et grande charge initiale, la surpression s'annule progressivement sans jamais changer de signes (voir fig. 10 a).

$$
\text { a) } \quad P_{\lambda_{0}} \approx 1 \quad r=0
$$

C'est le cas d'une conduite de longueur moyenne

$$
\text { b) } \begin{aligned}
0<+<T_{r} & & \Phi_{\lambda \sigma}=-\rho \\
& >T_{r} & \Phi_{\lambda \sigma}=0
\end{aligned}
$$

Le coup de bélier se réduit à une seule impulsion (voir fig. $10 \mathrm{~b}$ ).

$$
\text { c) } 0<p_{\lambda_{0}}<1 \quad 1<r<0
$$

C'est le cas d'une conduite à grande hauteur de chute, à faible charge initiale : la surpression oscille avec la période $2 T_{r}$ (fig. 10c).

L'amplitude maximale de la surpression est obtenue dans les trois cas pendant la première période, et est proportionnelle à

$$
\Phi_{\lambda \sigma_{\max }}=\rho(1-r)
$$

\section{3. - Comparaison avec le résultat obtenu à} I'aide de la méthode d'Alliévi ( $\left.{ }^{1}\right)$. - La première équation du système fondamental d'Alliévi est la suivante:

$$
\begin{gathered}
\zeta_{1}^{2}-1=2 \rho\left(1-\eta_{1} \zeta_{1}\right) \\
\text { avec } \zeta_{1}^{2}=\frac{H_{1}}{H_{0}}=\begin{array}{l}
\text { valeur relative de la pression } \\
\text { pendant le temps compris } \\
\text { entre } O \text { et } T_{r}
\end{array} \\
\eta=\begin{array}{l}
\eta \text { valeur relative de l'ouverture. } \\
\sigma=\zeta_{1}^{2}-1=
\end{array}
\end{gathered}
$$

(1) Voir article du Prof. Dr H. FAVRE - «Les coups de bélier d'ouverture brusque dans les conduites à caractéristiques linéairement variables. "Bullet. Techn. de la Suisse Romande - 15 septembre 1945.
Nous calculons $\sigma_{1}$

$$
\sigma_{1}=2 \rho\left(\rho \eta^{2}+1-\eta \sqrt{(\rho \eta)^{2}+2 p+1}\right)
$$

Nous admettons que $n_{1}=1+\lambda$ c'est-àdire que au temps $t=0$ l'obturateur a été varié brusquement de la valeur relative $\lambda$ à partir de la pleine ouverture. Nous différencions par rapport à $n$ et posons $\mathrm{d} n=\lambda$

$$
\begin{aligned}
& \sigma=2 \rho \lambda-\lambda \sqrt{(\rho \eta)^{2}+2 \rho+1}-\frac{\lambda^{2}}{\sqrt{\rho \eta^{2}+2 p+1}} \\
& \sigma \sim 2 \rho \lambda\left(2 \rho-(\rho+1)-\frac{\rho^{2}}{\rho+1}\right)=2 \rho \lambda \frac{\rho^{2}-1-\rho^{2}}{\rho+1} \\
& \frac{\sigma}{\lambda} \sim-\frac{2 \rho}{\rho+1}
\end{aligned}
$$

ce qui correspond au résultat que nous avions déjà obtenu.

4. - Cas particulier d'une conduite à basse chure. - Pour une centrale à basse chute, l'équation différentielle du mouvement de l'eau prend une forme très simple ${ }^{(1)}$ en assimilant la différentielle de $x$ à la longueur totale de la conduite. Nous obtenons :

$$
\begin{gathered}
v_{m} \frac{\delta \frac{v}{v_{m}}}{\delta t}=\frac{g H_{0}}{L} \frac{H_{1}}{H_{0}} \\
\sigma=\frac{H_{1}}{H_{0}}=\frac{L v_{0}}{g H_{0}} \frac{\delta y}{\delta \dagger}=\theta \frac{\delta y}{\delta t} \\
\text { avec } \theta=\frac{L v_{m}}{g H_{0}}=\rho T_{r}=\text { temps caractéristi- }
\end{gathered}
$$
que de la conduite.

L'équation donnée par les conditions limites au début de la conduite prend la forme très simple suivante:

$$
\theta p \gamma=-\theta\left(\frac{\lambda_{0}}{2} p \sigma+p \lambda\right)=\sigma
$$

d'où nous calculons la fonction de transfert de la surpression

$$
\begin{aligned}
& \phi_{\lambda \sigma}=\frac{\sigma}{\lambda}=-\frac{2}{\lambda_{0}} \frac{p T_{c}}{1+p T_{c}} \\
& \text { ovec } T_{c}=\frac{\theta \lambda_{o}}{2}=\frac{\rho T_{r} \lambda_{o}}{2}
\end{aligned}
$$

constante de temps de la conduite.

(1) P. ALMERAS - La Houille Blanche - Novembre 1945, page 81. 
Nous appliquons la transformation de Laplace à cette fonction de transfert et obtenons la courbe de réponse $\Phi_{\lambda, \sigma}$ de la pression dans ce cas particulier :

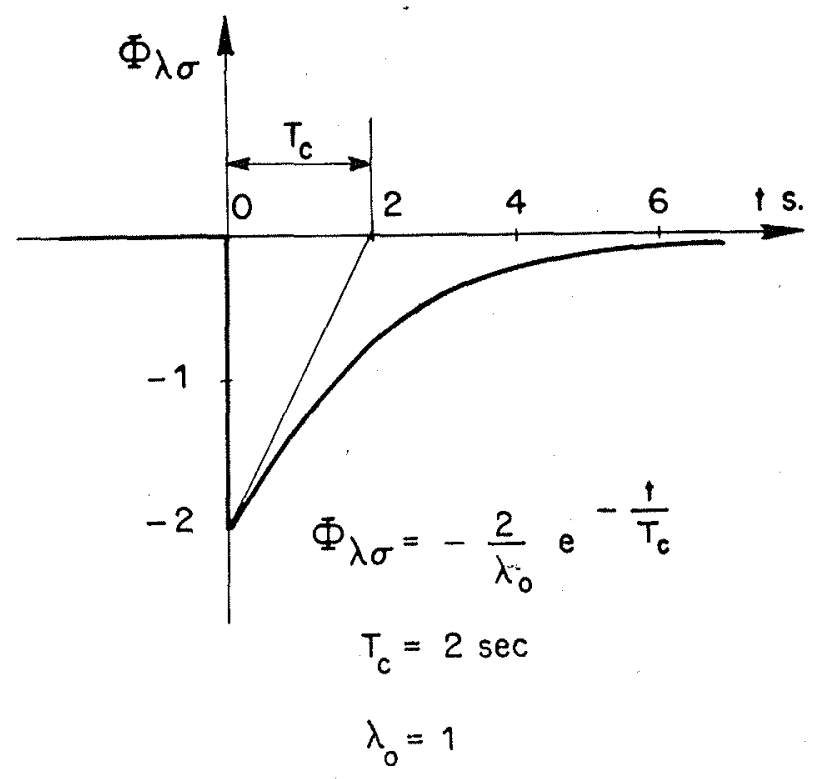

Fig. 11. - Courbe de réponse de la surpression pour une conduite de faible chute.

Ainsi que le représente la fig. 11, cette courbe de réponse est une exponentielle qui peut être considérée comme le cas limite de la fig. 10a lorsque le produit $\rho \hat{\lambda}$, devient infiniment grand.

Le même résultat peut être obtenu en posant $p \lambda_{0}=\infty$

dans l'expression générale :

$$
\Phi_{\lambda \sigma}=-\rho(1-r) r^{n}
$$

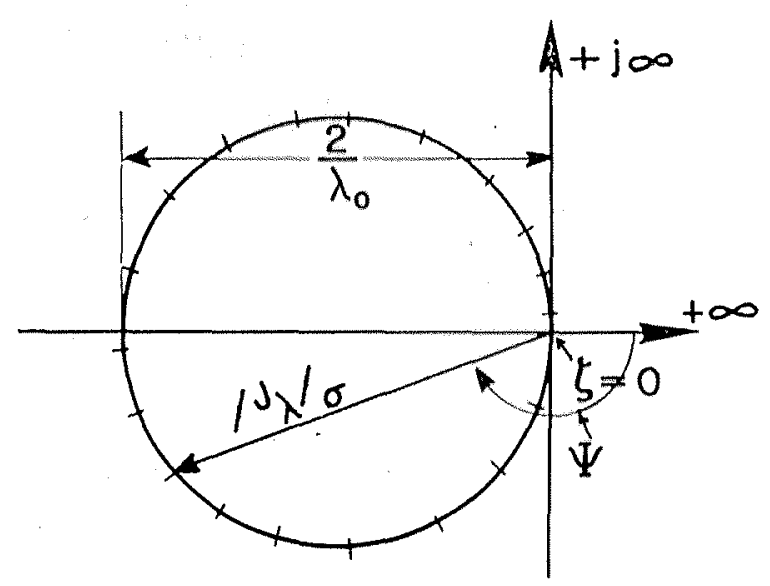

(a)
En effet, nous pouvons écrire :

$$
\begin{aligned}
& r=\frac{\rho \lambda_{0}-1}{\rho \lambda_{0}+1}=\left(1-\frac{1}{\rho \lambda_{0}}\right)\left(\frac{1}{1+\frac{1}{\rho \lambda_{0}}}\right) \simeq\left(1-\frac{1}{\rho \lambda_{0}}\right)^{2} \\
& r^{n}=\left(1-\frac{1}{\rho \lambda_{0}}\right)^{\frac{21}{T_{r}}}=\left(1-\frac{1}{\rho \lambda_{0}}\right)^{\rho \lambda_{0} \frac{1}{r_{c}}}
\end{aligned}
$$

11 est connu que

$$
\left.\lim \left(1+\frac{x}{n}\right)^{n}\right|_{n=\infty}-e^{x}
$$

donc

$$
\left.\left(1-\frac{1}{\rho \lambda_{0}}\right)^{\rho \lambda_{0}}\right|_{\rho \lambda_{0}=\infty}=\mathrm{e}^{-1}
$$

d'autre part

donc

$$
\rho(1-r)=\frac{2}{\lambda_{0}} \frac{\rho \lambda_{0}}{1+\rho \lambda_{0}}
$$

$$
\left.\rho(1-r)\right|_{\rho \lambda_{0=\infty}}=\frac{2}{\lambda_{0}}
$$

nous retrouvons donc bien

$$
\lim \Phi_{\lambda \sigma}=-\left.\rho(1-r) r^{n}\right|_{\rho \lambda_{0}=\infty}=-\frac{2}{\lambda_{0}} e^{-\frac{1}{T_{c}}}
$$

c) Courbe de Nyquist de la surpression. - II est aisé de calculer les surpressions qui résultent d'une oscillation harmonique de l'ouverture

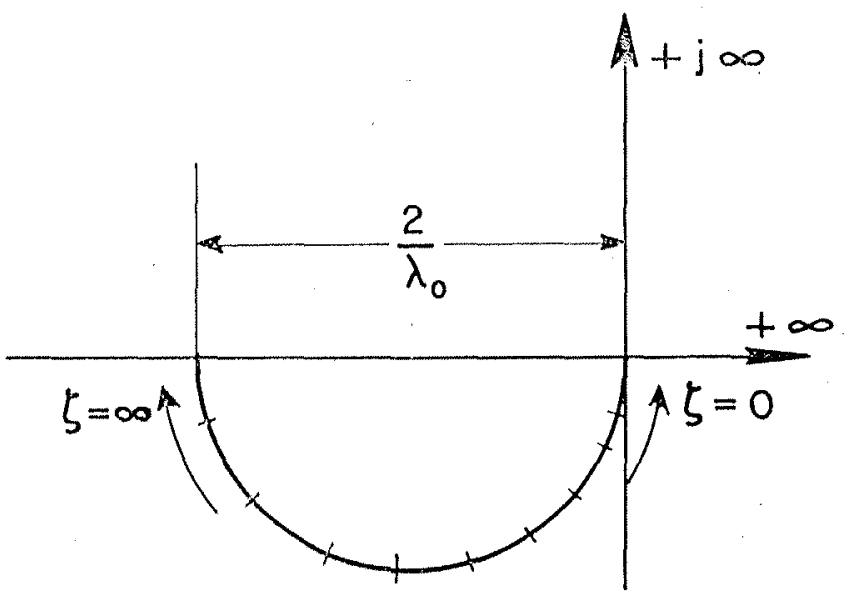

(b)

Fig. 12. - Courbe de Nyquist de la surpression. 
en remplaçant $p$ par $j \zeta$ la fonction de transfert.

$$
J_{\lambda \sigma}=-\frac{2 \rho}{\rho \lambda_{0}-j \operatorname{cotg} \frac{\zeta T_{r}}{2}}
$$

La fig. 12a montre que cette courbe de $\mathrm{Ny}$ quist est un cercle situé dans le deuxième et le troisième quadrant. Pour une pulsation $\zeta$ donnée, nous obtenons un déphasage :

et une valeur absolue :

$$
\Psi=-\operatorname{arctg} \frac{\operatorname{cotg} \zeta \frac{T_{r}}{2}}{\rho \lambda_{0}}
$$

$$
\left|J_{\lambda \sigma}\right|=\frac{2 \rho}{\sqrt{\left(\rho \lambda_{0}\right)^{2}+\left(\operatorname{cotg} \zeta \frac{T_{r}}{2}\right)^{2}}}=\frac{2}{\lambda_{0}} \cos \psi(1)
$$

Pour les fréquerices des oscillations telles que $\operatorname{cotg} \zeta \frac{\operatorname{Tr}}{2}=0$

de résonance;

l'oscillation de pression est en opposition de phase par rapport à celle de la variation de l'ouver. ture ; sa grandeur absolue est maximale, égale à $\frac{2}{\lambda_{0}}$. Pour de faibles charges, cette surpression relative peut prendre des valeurs considérables.

Par contre, pour les fréquences des oscillations telles que $\operatorname{cotg} \zeta \frac{\mathrm{Tr}}{2}=\infty$ la surpression est nulle.

La fig. 12b représente le cas d'une conduite de faible longueur. La courbe de Nyquist se ré-
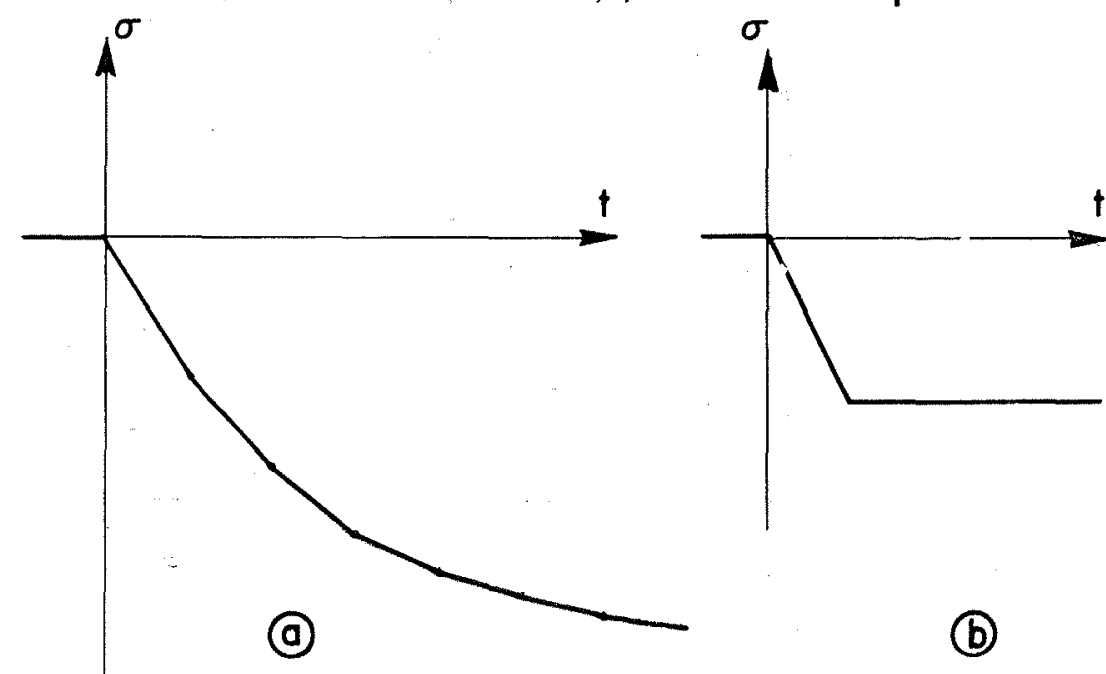

(b)

duit à un demi-cercle

$$
J_{\lambda \sigma}=-\frac{2}{\lambda_{0}} \frac{j \zeta T_{c}}{1+j \zeta T_{c}}
$$

Le calcul admettait que les variations de l'ouverture étaient faibles par rapport à la valeur initiale de l'ouverture, il n'est donc pas valable lorsque l'ouverture initiale est nulle $(?=0) \mathrm{ce}$ qui conduirait théoriquement à une surpression infinie, pour une conduite rigide $(0=\infty)$.

d) Surpression à la suite d'une variation quelconque de l'ouverture. - Soit ; ( t) la variation de l'ouverture et $\lambda$ (p) sa transformée de Laplace.

La valeur de la surpression sous forme opérationnelle est égale au produit de $\lambda$. (p) par.la fonction de transfert. 37.5

$$
\sigma(p)=\phi_{\lambda \sigma} \cdot \lambda(p)
$$

II suffit d'appliquer la transformation de Laplace opérateur-temps, pour calculer la surpression.

Considérons par exemple le cas d'une variation linéaire lente de l'ouverture :

\begin{tabular}{ccc}
$\lambda(t)=\frac{t}{T_{f}}$ \\
\hline$\rho \lambda_{0}>1$ & $\rho \lambda_{0}=1$ & $\rho \lambda_{0}<1$ \\
$\lambda_{0}=1$ & $\lambda_{0}=1$ & $\lambda_{0}=1$ \\
$\rho=5,7$ & $\rho=1$ & $\rho=0,38$ \\
$r=0,7$ & $r=0$ & $r=-0,4$
\end{tabular}
a)
b)
c)

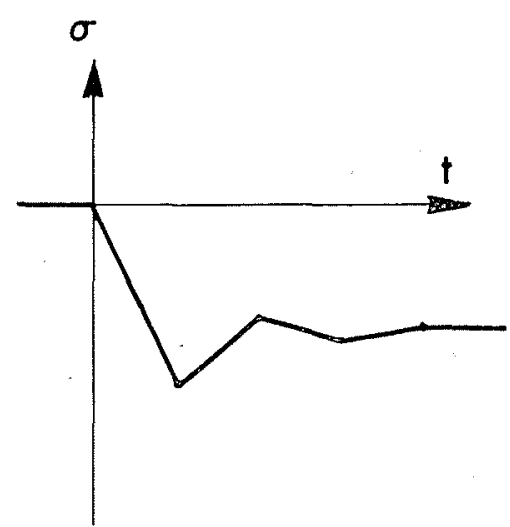

(C)

Fig. 13. - Surpression résultant d'une variation linéaire lente de l'ouverture.

(1) Ces valeurs correspondent bien à celles que donne M. Gaden aux pages 60 et 77 de son exposé : Etude de la stabilité d'un réglage automatique de vitesse par des diagrammes vectoriels. 
$T_{\mathrm{f}}=$ temps de fermeture.

La transformée de Laplace a la valeur suivante:

$$
\lambda(p)=\frac{1}{p T_{f}}
$$

nous obtenons :

$$
\sigma(p)=-\frac{2 \rho}{p T_{f}\left(\rho \lambda_{0}+\operatorname{coth} p \frac{T_{r}}{2}\right)}
$$

Nous pouvons déterminer en première approximation la surpression en considérant qu'une division par l'opérateur p correspond à intégrer par rapport au temps.

Suivant les valeurs du facteur de réflexion, nous obtenons par intégration-des courbes de la fig. 10 les courbes représentées par la fig. 13.

Pour le cas particulier d'une conduite à faible chute, nous obtenons la courbe exponentielle dessinée en pointillé sur la fig. 13a.
Pour l'usine de Soulom, nous obtenons les valeurs numériques suivantes:

$$
\begin{array}{lll}
H_{0}=252,50 \mathrm{~m} & a=1068^{\mathrm{m}} / \mathrm{s} & \lambda_{0}=1 \\
v=1,36 \mathrm{~m} / \mathrm{s} & \rho=0,294 & r=0,55
\end{array}
$$

La fig. 14 donne un contrôle qualificatif let quantitatif de la méthode de calcul que nous iridiquons, la comparaison des courbes 2 (surpression mesurée) avec les courbes 3 (surpression calculée d'après la méthode d'Alliévi) (1) et 4 (surpression calculée d'après la méhode ci-dessus décrite) est satisfaisante.

e) Conduites à caractéristriques multiples. En pratique, pour des raisons de sollicitation mécanique, les caractéristiques d'une conduite forcée ne sont pas constantes. Cependant, en preinière approximation, en négligeant les phénomènes de réflexion partielle aux discontinuités de la conduite, ce cas général peut être ramené

(1) Voir CAMICHEL EYDOUX et GARIEL : Etudes théoriques et expérimentales des coups de bélier, poge 149 figure 105.

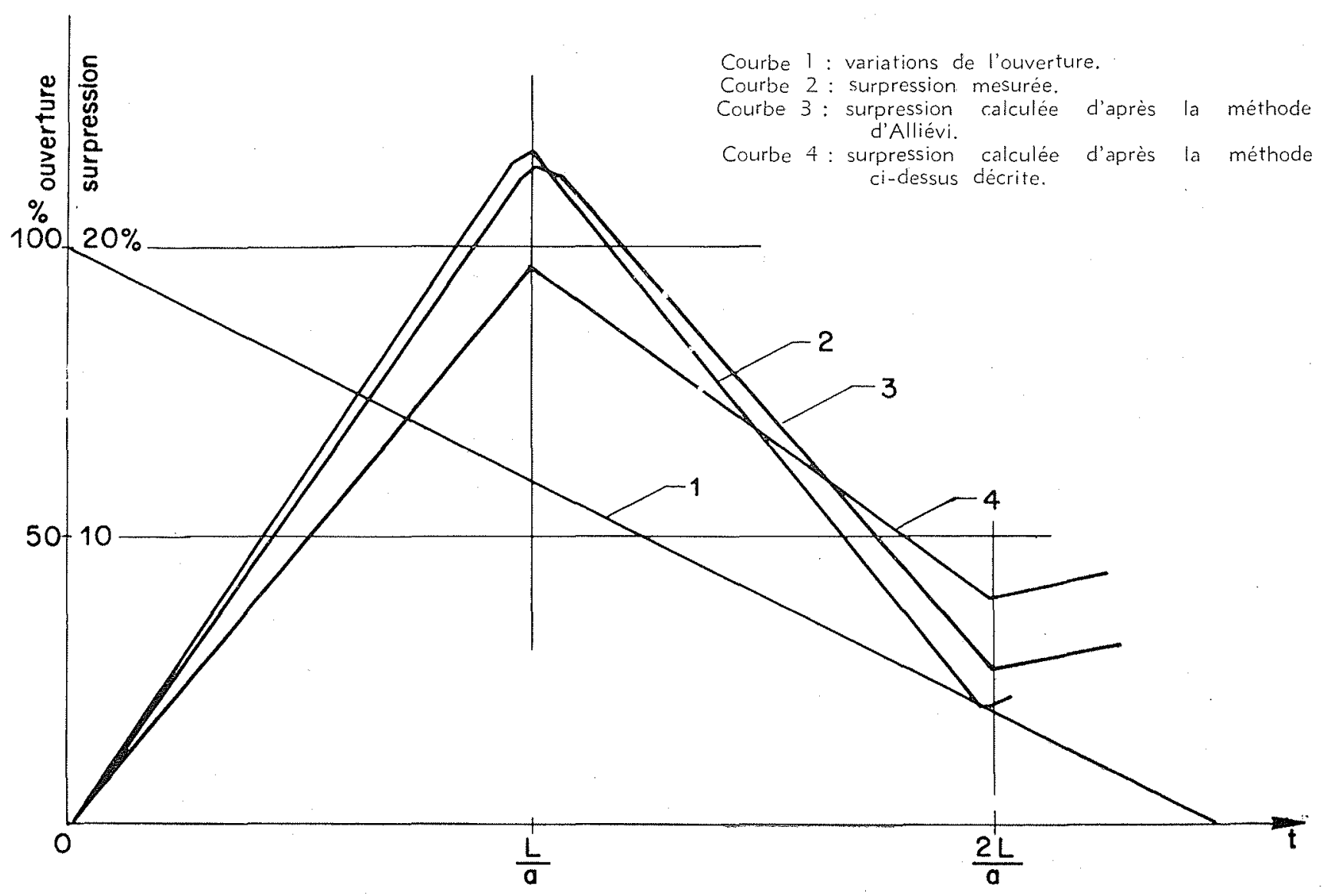

Fig. 14. - Comparaison entre surpression calculée et mesurée. 
au cas particulier d'une conduite à caractéristique unique, si l'on introduit les valeurs moyennes suivantes:

vitesse moyenne de l'eau:

vitesse moyenne de propaga-

tion des ondes de pression

Grandeur caractéristique de la conduite:

$$
\begin{aligned}
& v_{m}=\frac{Q}{L} \sum \frac{\Delta L_{i}}{S_{i}} \\
& a_{m}=\frac{L}{\sum \frac{\Delta L_{i}}{a_{i}}}
\end{aligned}
$$

$$
\rho=\frac{a_{m} v_{m}}{2 g H}
$$

avec $Q$ - débit en $\mathrm{m}^{3} / \mathrm{sec}$. à pleine ouverture. longueur des différents tronçons $\Delta \mathrm{L}_{\mathrm{i}}=\quad$ en $\mathrm{m}$.

i section des différents troncons en $s_{i}=m^{2}$.

$A_{i}=$ vitesse de propagation dans les dif férents tronçons.

\section{VI. - CARACTERISTIQUE DYNAMIQUE DES VARIATIONS DE PUISSANCE DE LA TURBINE.}

a) Fonction de transfert des variations de puissance de la turbine. - Nous introduisons la valeur de la fonction de transfert de la pression dans l'expression de la fonction de transfert ide la puissance de la turbine que nous avons déjà déterminée.

$$
\phi_{\lambda \mu_{t}}=1+\frac{3}{2} \lambda_{0} \phi_{\lambda \sigma}=1-\frac{3 \rho \lambda_{0}}{\rho \lambda_{0}+\operatorname{coth} \rho \frac{T_{r}}{2}}
$$

Pour le cas particulier d'une conduite à faible chute, nous obtenors :

$$
\phi_{\lambda \mu_{t}}=1-\frac{3 p T_{c}}{1+p T_{c}}
$$

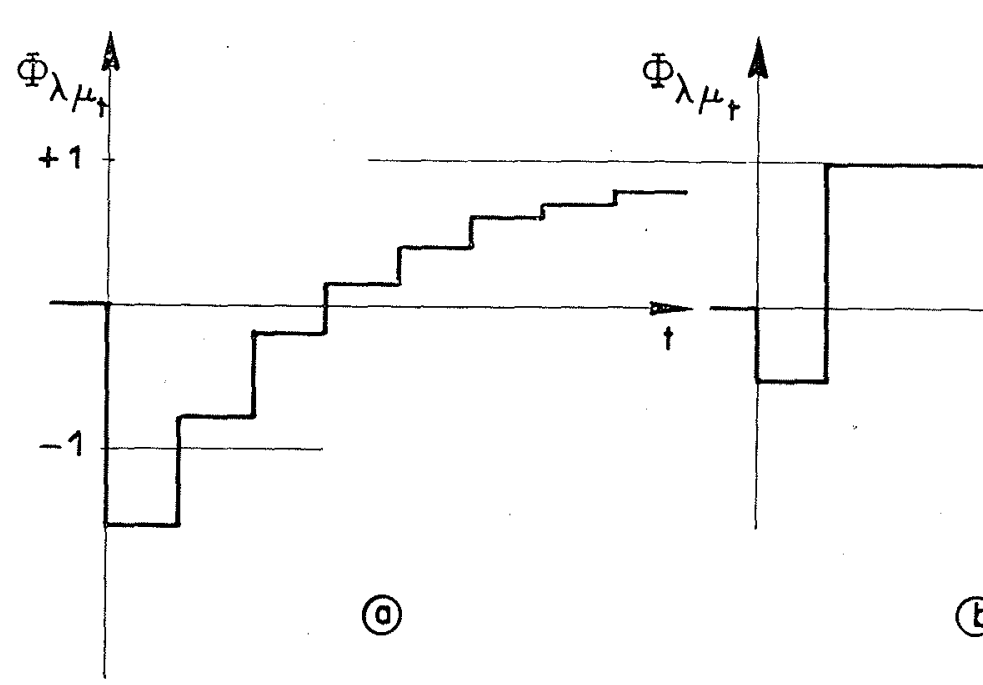

b) Courbe de réponse des variations de puissance de la turbine. - En première approximation, nous obtenons:

pour

$$
\begin{aligned}
& n T_{r}<t<(n+1) T_{r} \\
& \Phi_{\lambda_{\mu_{t}}}=1-\frac{3}{2}(1+r) r^{n}
\end{aligned}
$$

la fig. 15 représente cette courbe de réponse pour différentes valeurs du facteur de réflexion. Il est intéressant de constater que cette courbe de réponse ne dépend que de $r$ et est indépendante de la charge initiale $\lambda_{0}$.

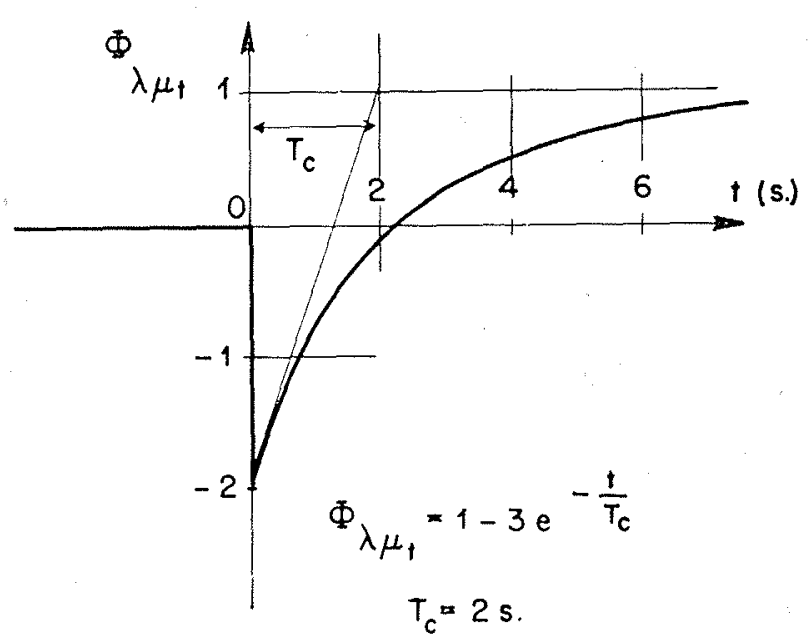

Fig. 16. - Courbe de réponse pour une conduite de faible chute.

La fig. 16 représente cette courbe de réponse dans le cas d'une conduite à faible chute.

c) Courbe de Nyquist des variations de puissance de la turbine. - A partir de la fonction de transfert, nous déduisons l'expression de la courbe d'allure en remplaçant $p$ par i's.

Fig. 15. - Courbe de réponse des variations de puissance de la turbine pour différentes valeurs du facteur de réflexion. 


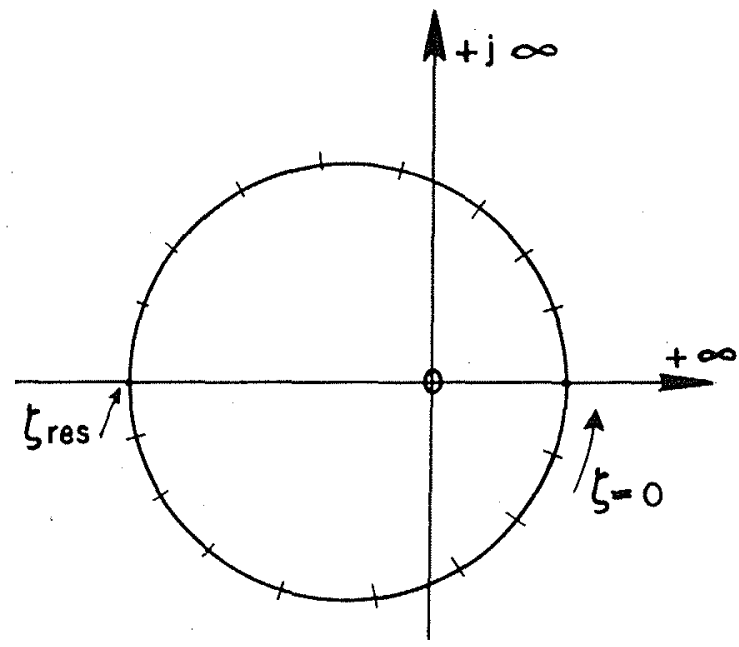

(a)

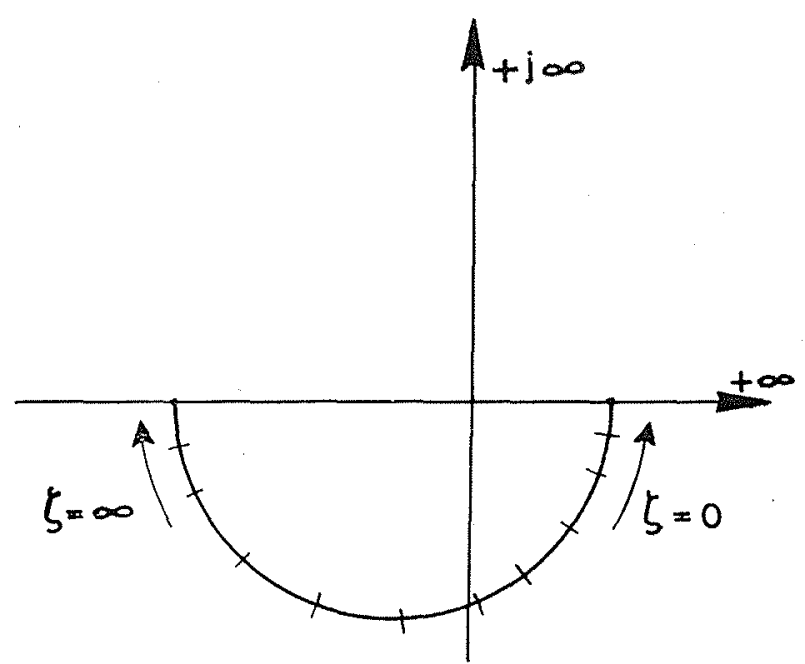

(b)

Fig. 17. - Courbe de Nyquist de la puissance de la turbine.

a) haute et moyenne chute

b) basse chute

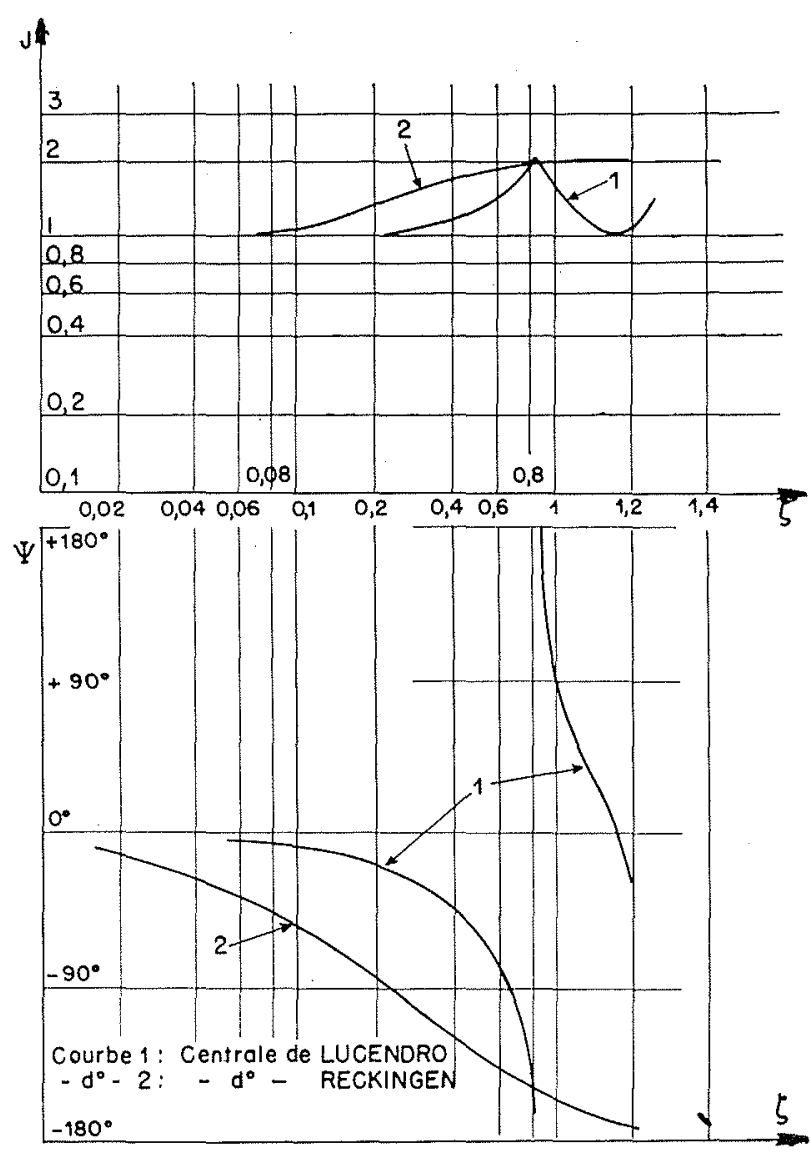

Fig. 18. - Courbe de Nyquist de la puissance de la turbine sous forme cartésienne.
Ainsi que le représente la fig. 17a, la courbe de Nyquist :

$$
J_{\lambda \mu_{f}}=1-\frac{3 \rho \lambda_{0}}{\rho \lambda_{0}-j \operatorname{cotg} \frac{\zeta T_{T}}{2}}
$$

est un cercle ayant un diamètre égal à 3 , et son centre à la cote $(-0,5 ;$ jo $)$; la forme de cette courbe de Nyquist est la même, quels que soient $\rho \lambda_{0}$ et $T_{r}$, seules les cotes changent ; la courbe de Nyquist pour une conduite à faible chute:

$$
J_{\lambda \mu_{t}}=1-3 \frac{1 \zeta T_{c}}{1+j \zeta T_{c}}
$$

Est représentée par la fig. 17b, le cercle dégénère en un demi-cercle.

La fig. 18 représente ces mêmes courbes. de Nyquist, sous forme cartésienne pour les valeurs numériques correspondant au cas particulier des centrales de Lucendro et de Reckingen en Suisse.

\section{VII. - CARACTERISTIQUE DYNAMIQUE DES VARIATIONS DE VITESSE.}

a) Fonction de transfert des variations de vitesse. - Nous considérons le cas particulier du groupe dont les conditions de marche sont telles que les puissances électrique et mécanique sont indépendantes de la vitesse, ce qui est un cas défavorable en ce qui concerne la stabilité du réglage de vitesse. 
En introduisant la valeur de la fonction de transfert pour la puissance de la turbine dans l'expression de la fonction de transfert pour la vitesse, nous obtenons pour la conduite de grande et de moyenne chutes :

$$
\phi_{\lambda \nu}=\frac{1}{p T_{a}}\left(1-\frac{3 p \lambda_{0}}{\rho \lambda_{0}+\operatorname{cotg} p \frac{T_{r}}{2}}\right)
$$

pour la conduite à faible chute :

$$
\phi_{\lambda \nu}=\frac{1}{p T_{a}}\left(1-\frac{3 p T_{c}}{1+p T_{c}}\right)
$$

b) Courbe de réponse des variations de vitesse. - La courbe de réponse peut se calculer en appliquant la formule de Heaviside à la fonction de transfert.

Selon la fig. 19, une première approximation peut être obtenue en intégrañt les courbes de réponse de la fig. 15.

Pour une conduite de faible chute, la fonction de transfert $\Phi \bar{y}$, se calcule aisément à partir de l'expression de la fonction de transfert

$$
\Phi_{\nu \lambda}=\frac{t}{T_{a}}-\frac{3 T_{c}}{T_{a}}\left(1-e^{-\frac{t}{T_{c}}}\right)
$$

et se trouve représentée par la fig. 20.

C) Courbe de Nyquist des variations de vitesse. - La courbe de Nyquist se déduit à nouveau en remplaçant l'opérateur $p$ par $j \zeta$ dans l'expression de la fonction de transfert. La courbe de Nyquist J Jiy sous forme cartésienne pour le cas particulier de Lucendro est représentée :par les courbes $J_{i y}$ et $\Phi_{\lambda, y}$ de la fig. 21 .

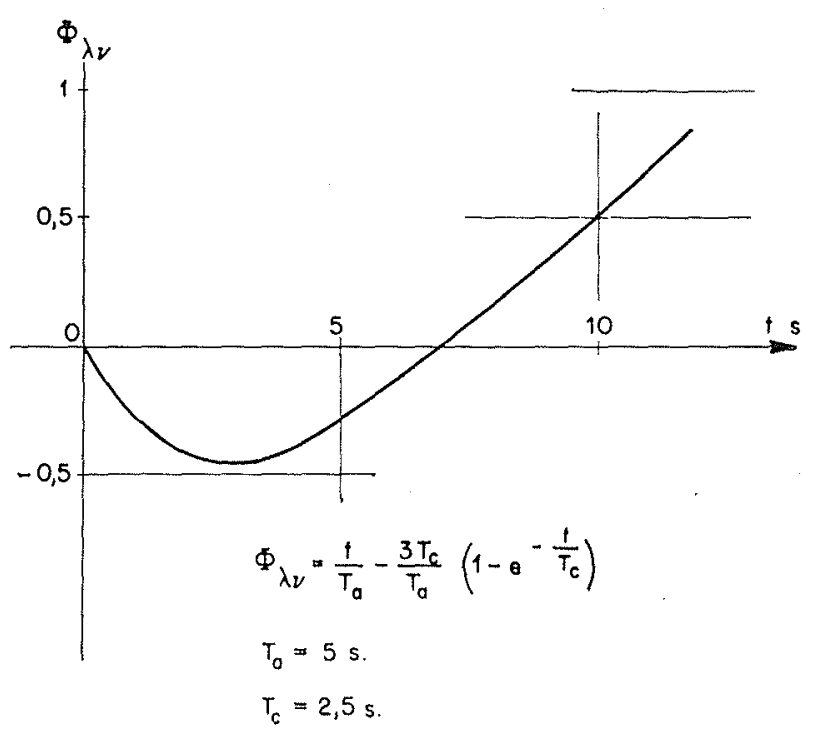

Fig. 20. - Courbe de réponse de la vitesse pour une conduite de faible chute.

\section{VIII. - DETERMINATION DES CONDITIONS DE STABILITE.}

A titre d'exemple, nous avons choisi un réglage correspondant à celui de l'un des groupes de la centrale de Lucendro en prenant la constante d'accélération $T_{a}$ c'est-à-dire le $\mathrm{PD}^{2}$ comme paramètre. Lorsque les courbes d'allure du dispositif de réglage et du groupe sont déterminées, il est aisé de déterminer graphiquement la courbe

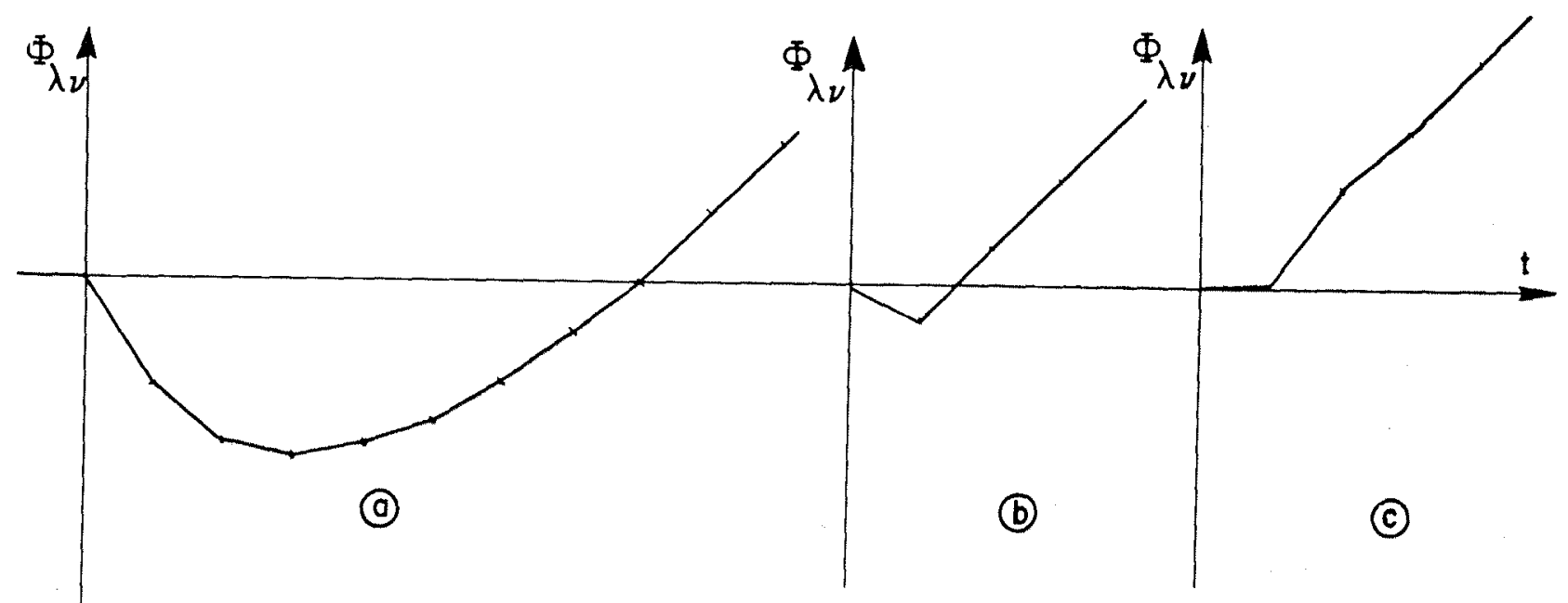

Fig. 19. - Courbe de réponse de la vitesse. 
d'allure du réglage ouvert $J_{k}$ ainsi que le représente la fig. 21.

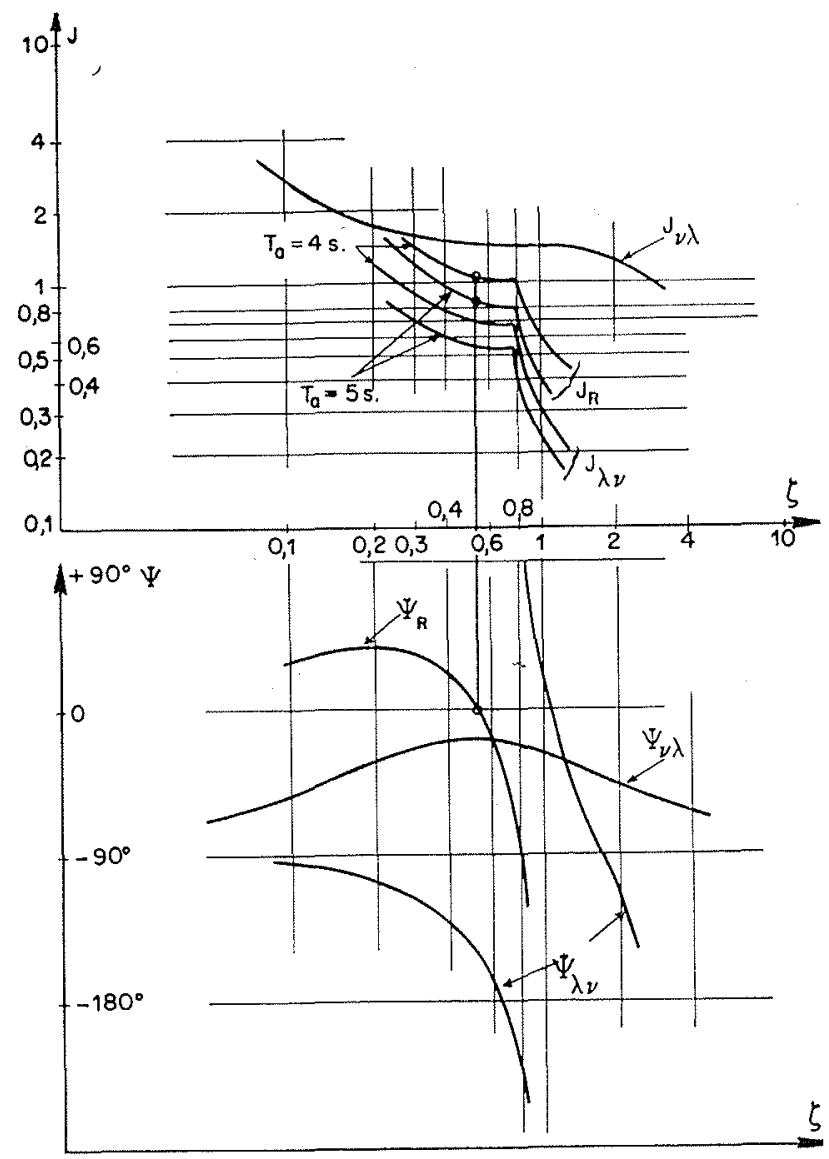

Fig. 21. - Courbe de Nyquist de rég'age sius forme cartésienne.

1) Courbe de Nyquist du dispositif de réglage Jik, 世in.

2) Courbe de Nyquist du groupe $J_{\lambda y}, \Psi_{i, \%}$

3) Courbe de Nyquist du circuit de réglage $J_{11} \Psi_{n}$

Nous voyons qu'en admettant les conditions défavorables des puissances de la turbine et du générateur indépendantes de la vitesse, le groupe est stable pour $T_{i} \geq 5 \mathrm{~s}$.

instable pour $T u \leqslant 4 s$.

Ce groupe a été exécuté avec un $\mathrm{PD}^{2}$ tel que $\mathrm{T}^{\mathrm{i}}=5,2 \mathrm{~s}$.

En première approximation, il est légitime de faire le calcul en admettant que le dispositif de réglage est purement statique avec un statisme égal au statisme passager. Nous obtenons les formules suivantes :

Centrale à haute et moyenne chute :

$$
T_{a} \delta>\frac{\sqrt{2}}{\zeta_{\text {res }}} \text { avec tg } \zeta_{\text {res }} \frac{T_{r}}{2}=\frac{1}{\sqrt{2} \lambda_{o} \rho}
$$

avec

$\zeta_{\text {ris }}=$ pulsation pour laquelle le déphasage de la courbe d'allure de réglage est nul.

\section{Centrale à haute et moyenne chute :}

$$
\mathrm{T}_{\mathrm{\alpha}} \delta>2 \mathrm{~T}_{\mathrm{s}}
$$

\section{IX. - ANALOGIE ELECTRIQUe.}

Les équations différentielles qui décrivent les phénomènes de propagation d'une onde électromagnétique dans un câble homogène sont du même type que celles qui régissent les mouvements de l'eau dans une conduite forcée, si l'on idelitirie la pression hydraulique à la tension électrique et la vitesse de l'eau au courant électrique.

Soit un câble supposé sans perte alimenté par une tension $E$ sur une résistance $R$ et courtcirclité en son autre extrémité, tel que le représente la fig. 22.

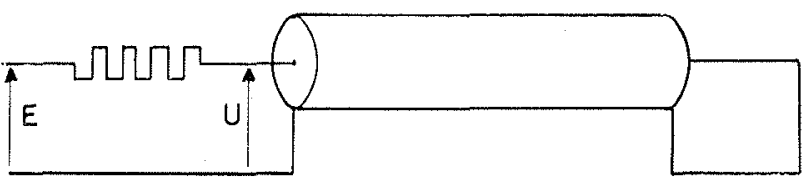

Fig. 22. - Modèle électrique d'une conduite forcée.

L'expression de la tension d'entrée $U$ en fonction de la tension E écrite sous forme opérationnelle a la forme suivante (1).

$$
\frac{U}{E}=\frac{Z}{R} \frac{1}{\frac{Z}{R}+1} \frac{1-e^{-\frac{2 L}{w}}}{q-r e^{-p \frac{2 L}{w}}}
$$

avec $Z=$ impédance caractéristique $=\sqrt{\frac{l_{s}}{c_{s}}}$

$w=$ vitesse de propagation $=\frac{1}{\sqrt{I_{s} \mathrm{c}^{0}}}$

$\mathrm{I}_{\mathrm{s}}=$ inductivité spécifique

$c_{\mathrm{s}}=$ capacité spécifique

$L=$ longueur du câble

$$
r=\frac{Z-R}{Z+R}=\frac{\frac{Z}{R}-1}{\frac{Z}{R}+1}
$$

en identifiant $\frac{R}{z}$ et $\lambda_{0} \rho$

$$
w \text { et a }
$$

\section{.}

nous retrouvons l'expression de la fonction de transfert de la surpression, ce qui laisse envisager le parti qu'il y aurait à tirer d'un modèle électri-

(1) Voir K.-W. WAGNER, Operatorenrechnung, page 98. 
que, soit pour l'étude des phénomènes des coups de bélier dans une conduite à caractéristiques multiples, soit pour l'étude générale des conditions de stabilité d'un réglage de vitesse; il est en effet aisé de déterminer un modèle électrique pour les autres éléments du circuit de réglage. La combinaison de ces différents modèles permet de vérifier expérimentalement de façon commode les résultats auxquels conduirait une étude théorique, en particulier pour la détermination des modes de stabilisation.

Le cas d'une conduite infiniment courte serait représenté par un câble qui se réduirait à une self.

\section{X. - CONCLUSION.}

Si l'établissement du critère de Nyquist fait appel à de hautes mathématiques, son utilisation, par contre, n'offre aucune difficulté de calcul puisqu'elle est graphique.

La signification physique des courbes de réponse et des courbes de Nyquist, la possibilité de les relever expérimentalement et de les contrôler, voire de supprimer tout calcul, doit leur attirer la faveur des praticiens.

Les développements que nous indiquons traitent le phénomène du coup de bélier dans sa généralité, c'est-à-dire aussi bien pour les centrales à faible chute que pour les centrales à moyenne et à haute chute. Ils déterminent les variations de la pression, du couple moteur et de la vitesse résultant d'une brusque variation du vannage. Ces « courbes de réponse » donnent une image très concrète de la répercussion des coups de bélier sur le réglage de vitesse des turbines hydrauliques. Crâce à l'utilisation des transformations de Laplace, ii est aisé de déterminer les "courbes de Nyquist » correspondantes et de définir les conditions de stabilité avec un minimum de calcul, en appliquant le critère de $\mathrm{Ny}$ quist. Cette méthode permet de tenir compte de certains facteurs que la méthode classique devait négliger pour ne pas trop compliquer les calculs, tels que les retards introduits par les jeux du dispositif de réglage, ou les phénomènes de réso- nance qui peuvent se produire pour une centrale à chute moyenne accouplée faiblement à un puissant réseau, les fréquences propres de la conduite et du générateur pouvant être du même ordre de grandeur.

Pour le cas particulier d'un dispositif de réglage infiniment rapide et purement statique, nous donnons des formules très simples qui donnent une première approximation de la relation 'qui existe entre la constante d'accélération, c'est-àdire le $\mathrm{PD}^{2}$ à donner au groupe, les caractéristiques hydrauliques du système d'alimentation de la turbine et le statisme passager ou le dosage accélérométrique à donner au dispositif de réglage. Ces conditions rejoignent celles que $M$. ALMERAS indique pour les centrales à basse chute.

Les calculs que nous indiquons reposent sur I'hypothèse que la vitesse d'écoulement de l'eau est négligeable par rapport à la vitesse de propagation des ondes de pression. Cette hypothèse n'est plus admissible par exemple pour l'étude de la stabilité d'une centrale établie à l'extrémité d'un canal d'amenée. II reste donc à les généraliser dans ce sens.

La méthode de calcul que nous employons fait en quelque sorte le pont entre les méthodes graphiques et semi-graphiques d'ALLIEVI et de SCHNYDER-BERGERON, applicables pour des courses de l'obturateur de la turbine de grandeur finie, et ne permettant pas de tirer des conclusions immédiates sur l'influence du coup de bélier sur le réglage de vitesse de la turbine, et d'autre part la méthode de calcul analytique à l'aide du critère de Hurwitz employée en particulier par MM. ALMERAS, GADEN et STEIN, qui ne permet pas de tenir compte directement de l'influence de la compressibilité de l'eau et de l'élasticité des parois de la conduite. Cependant ainsi que cela ressort de la comparaison des fig. 10 a et 11 , ainsi que 15 a et 16 , la concordance du résultat auquel conduisent ces deux dernières méthodes est excellente pour les centrales à basse chute (avec un coefficient e élevé). Comme ce cas représente les conditions les plus défavorables en ce qui concerne la stabilité du réglage de vitesse, les conclusions auxquelles les auteurs précités arrivaient s'en trouvent confirmées. 